Original Research Paper

\title{
Stochastic and Deterministic Mathematical Modeling and Simulation to Evaluate the Novel COVID-19 Pandemic Control Measures
}

\author{
${ }^{1}$ Eva Gupta, ${ }^{2 *}$ Nand Jee Kanu, ${ }^{3}$ Amartya Munot, \\ ${ }^{4}$ Venkateshwara Sutar, ${ }^{1}$ Umesh Kumar Vates and ${ }^{5}$ Gyanendra Kumar Singh \\ ${ }^{1}$ Amity University, Noida, India \& AGCE, Satara, India \\ ${ }^{2} S$. V. National Institute of Technology, Surat, India \& JSPM Narhe Technical Campus, Pune, India \\ ${ }^{3}$ S. A. P. D. J. Pathshala's Walchand Institute of Technology, Solapur, India \\ ${ }^{4}$ JSPM Narhe Technical Campus, Pune, India \\ ${ }^{5}$ Adama Science and Technology University, Adama, Ethiopia
}

Article history

Received: $12-10-2020$

Revised: 12-11-2020

Accepted: $19-11-2020$

Corresponding Author:

Nand Jee Kanu

S. V. National Institute of

Technology, Surat, 395007,

India \& JSPM Narhe Technical

Campus, Pune, 411041, India

Email: nandssm@gmail.com

\section{Abstract:}

Background: In India, the novel Coronavirus Disease (COVID-19) epidemic has grown to 17,00000 cases and around 38,000 deaths up to 30th July, 2020. The impacts of the Severe Acute Respiratory Syndrome Coronavirus 2 (SARS-CoV-2) pandemic in India were studied using modified agestructured stochastic and deterministic mathematical models.

Methods: A compartmental susceptible (S)-infected (symptomatic) $\left(I_{S}\right)$ infected (asymptomatic) $\left(I_{N}\right)$ recovered $(R)$ i.e., $S I_{S} I_{N} R$ model is developed, in which the flow of individuals through compartments is modeled using a set of differential equations. The outbreak of the novel COVID-19 pandemic is critically evaluated from all major angles using base, education, vaccination and education and vaccination models based on the modified $S I_{S} I_{N} R$ transmission network model for their simulations in MATLAB and peak of infected cases (both symptomatic and asymptomatic cases) as well as end of pandemic is predicted in each case.

Result: The numerical investigations are done for both stochastic and deterministic studies using the modified $S I_{S} I_{N} R$ transmission network model for effective prediction about the transmission of SARS-CoV-2 pandemic in India. The progress of the novel COVID-19 pandemic in India is estimated for various scenarios by varying the basic reproduction numbers from mean to extremes (general assumptions and strategies are inculcated through contact tracing based on the values of contact ratio operated for the basic reproduction numbers, ' $R_{0}$ '). The efficacy and potential of the education programs and vaccination programs were established with the published datasets through a validation studies. Furthermore, the outbreak of the novel COVID-19 pandemic is predicted for majorly affected cities in India on the basis of different reproduction numbers ' $R_{0}$ '.

Conclusion: The report presented herewith could be referred to revise the government policies to (a) implement mitigatory measures such as the practice of social distancing, partial city lockdown across the nation, etc., (b) implement a $100 \%$ daily number of tests to the susceptible population of the nation, (c) improve hospital facilities and the novel COVID-19 wards, (d) improve the recovery rate with the effective implementation of base, education, vaccination and education and vaccination model to attain the equilibrium stage of 
pandemic at the earliest and (e) meet the objective of preventing the outbreak of the novel COVID-19 pandemic through the effective implementation of control and prevention strategies across the nation.

Keywords: COVID-19, SARS-CoV-2, Pandemic, Mathematical Model, Education, Vaccination

\section{Introduction}

The novel COVID-19 pandemic has presented a prodigious ultimatum before the whole world, with that tardy response from the WHO that resulted bedlam in the subcontinent of India and most of the other countries in the world. As of 30th July, 2020, there have been $18,000,000$ confirmed cases of the novel COVID-19 and about 7,00000 reported deaths globally and, due to the rising economical unbalance, probably the countries around the world had to open financial markets. Due to which, there has been a great rise in the number of cases since the opening of the global market as there is no specific medication or vaccine approved by global medical authorities. The disease is transmitted by inhalation or contact with infected droplets or fomites and the incubation period during these days may range from 10-20 days. The disease can be fatal to elderly people and those with an underlying medical history (COVID-19 India, 2020; Hindustan Times, 2020a).

\section{What is Already known on the Subject}

The transmissibility of the disease outbreak is studied and it is observed that the strictness with which social distancing in India is followed, is insufficient to break the chain of the novel COVID-19. The impacts of the Severe Acute Respiratory Syndrome Coronavirus 2 (SARS-CoV-2) pandemic in India were studied using modified age-structured stochastic and deterministic mathematical models.

\section{What does this Study Add}

The major goal of the present investigation is to study the transmission of the novel COVID-19 pandemic in India for various possible scenarios based on general assumptions and strategies inculcated through contact tracing using the values of contact ratios which are operated for the basic reproduction numbers, ' $R_{0}$ ', with the aim to educate individuals to reduce the spread of the novel COVID-19 and vaccination program to assess their potential results in upcoming days.

The outbreak of the novel COVID-19 pandemic is critically evaluated from all major angles using base, education, vaccination and education and vaccination models based on the modified $S I_{S} I_{N} R$ model.

The outspread of any epidemic depends on the infectivity of the pathogen and the available population that is considered as susceptible. For a novel pandemic, when the spread of the disease is not clear yet, here the mathematical modeling estimates the number of cases in the worst and the best cases. It can also aid to estimate the effect of preventive measures that are been applied against the novel COVID-19. By using the suppressive strategy, the major objective is to reduce the basic reproduction number, ' $R_{0}{ }^{\prime}$ and maintain it below ' 1 '. To prohibit further infection spread using mitigatory measures; the main objective is to contain the spread and reduce the effect of the pandemic. The novel COVID-19 pandemic is caused by the Severe Acute Respiratory Syndrome Coronavirus 2 (SARSCoV-2). On 30th January, 2020, India witnessed its first case of the novel COVID-19. Currently, India is one of the most affected countries and has the highest number of cases in Asia continent. In the world, India was the third highest affected country after United States of America and Brazil, with over 100,000 confirmed cases on 19th May, 2020 and about 1,000,000 confirmed cases on 17th July, 2020. Whereas, in India the fatality rate is probably the lowest in the world at $2.11 \%$ as of 02 nd August, 2020 and it is also steadily reducing with time. With that, the six cities - Delhi, Ahmedabad, Chennai, Mumbai, Pune and Kolkata have around $50 \%$ of the total cases reported in the country. By the 24th May, 2020, Lakshadweep was the only region that was unaffected by the novel COVID-19 pandemic. The recovery rate in India is $64.23 \%$ as on 02nd August, 2020 (COVID-19 India, 2020; Hindustan Times, 2020a; Wikipedia, 2020a).

In India, the speed of testing is raised from June, 2020 as shown in Fig. 1 and up to 31st May, 2020, a strict lockdown has been imposed across the nation by the government as mitigatory measures to restrict the spread of the pandemic. On 09th April, 2020, Indian Council of Medical Research (ICMR) further revised the testing strategy and allowed testing of people showing symptoms for a week in the hotspot areas of the country, regardless of travel history or local contact to the patient. There has been a rise in the number of cases in India during lockdown as due to the religious gathering in the country's capital territory, Delhi. The Government of India announced in the press release on 18th April, 2020, that 4,291 cases, which are about $29.8 \%$ of the total 14,378 confirmed cases of the novel COVID-19 in India, had connection with religious and social gatherings. These cases contributed to spread of the pandemic across 23 states and few of the nation's union territories. By 04th April, 2020, nearly about 22,000 people that were 
in contact with the people who attended the social event were quarantined. In addition to that, pilgrims from Maharashtra state were sent by the state government back to Punjab. The total 609 of the around 4,000 stranded pilgrims who returned to Punjab from Nanded (a historical city in Maharashtra) were tested positive for the novel COVID-19, taking the state's infection tally to over 1,000 (Wikipedia, 2020a; Hindustan Times, 2020b).

The Indian government imposed the strict lockdown across the country to contain the spread of SARS-CoV-2 and built healthcare infrastructure to treat those who were affected. The lockdown was aggressive but vital. India's fast response to the pandemic was praised as comprehensive and robust by the United Nations (UN) as well as the World Health Organization (WHO). India has a vast experience in eradicating diseases like smallpox and polio. Owing to this, India is strong enough to deal with the outbreak of the novel COVID-19 as stated by Mr. Michael Ryan, (chief executive director of the WHO's health emergencies program). The lockdown was imposed by the government and as well as other agencies to contain the spread of SARS-CoV-2 and to help India to escape from the community transmission stage of the pandemic which could eventually make handling the situation very difficult and result in even more fatalities (Wikipedia, 2020a; Hindustan Times, 2020b; Livemint, 2020).

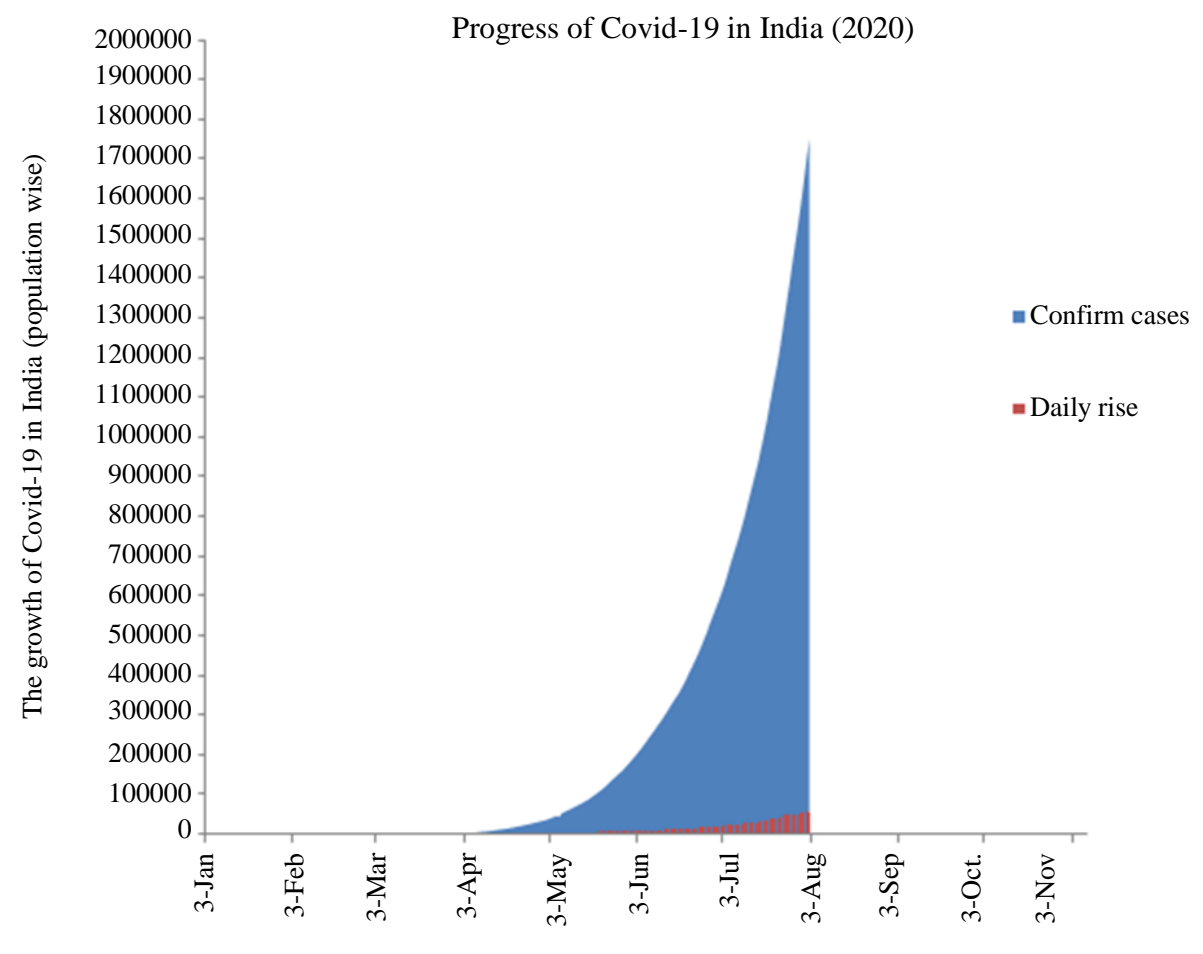

The outbreak of Covid-19 in India (month wise)

Fig. 1: Growth of the novel COVID-19 pandemic in India

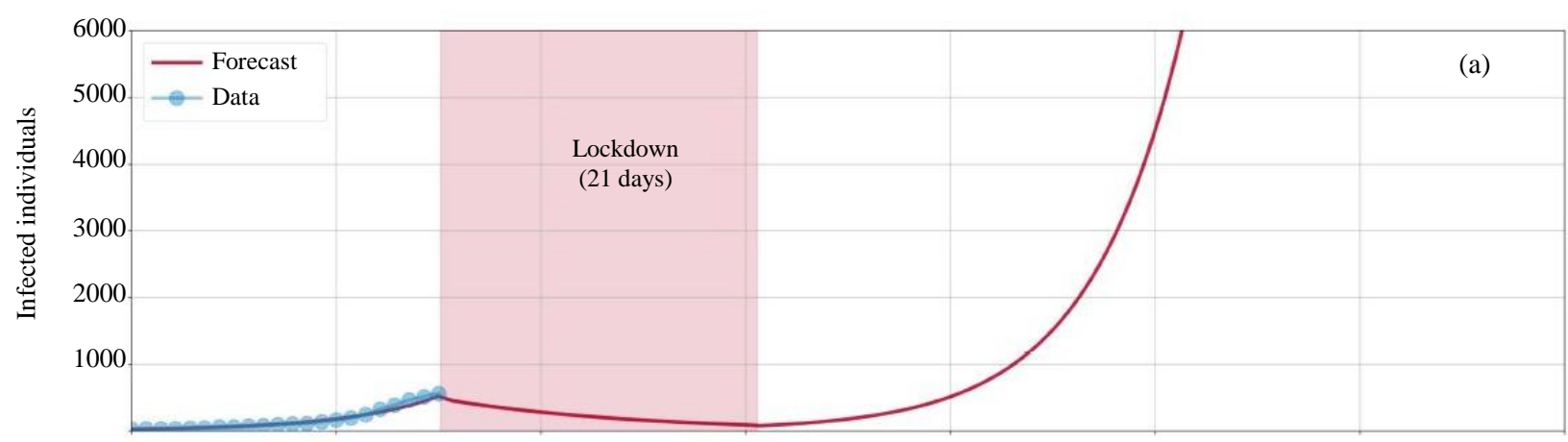



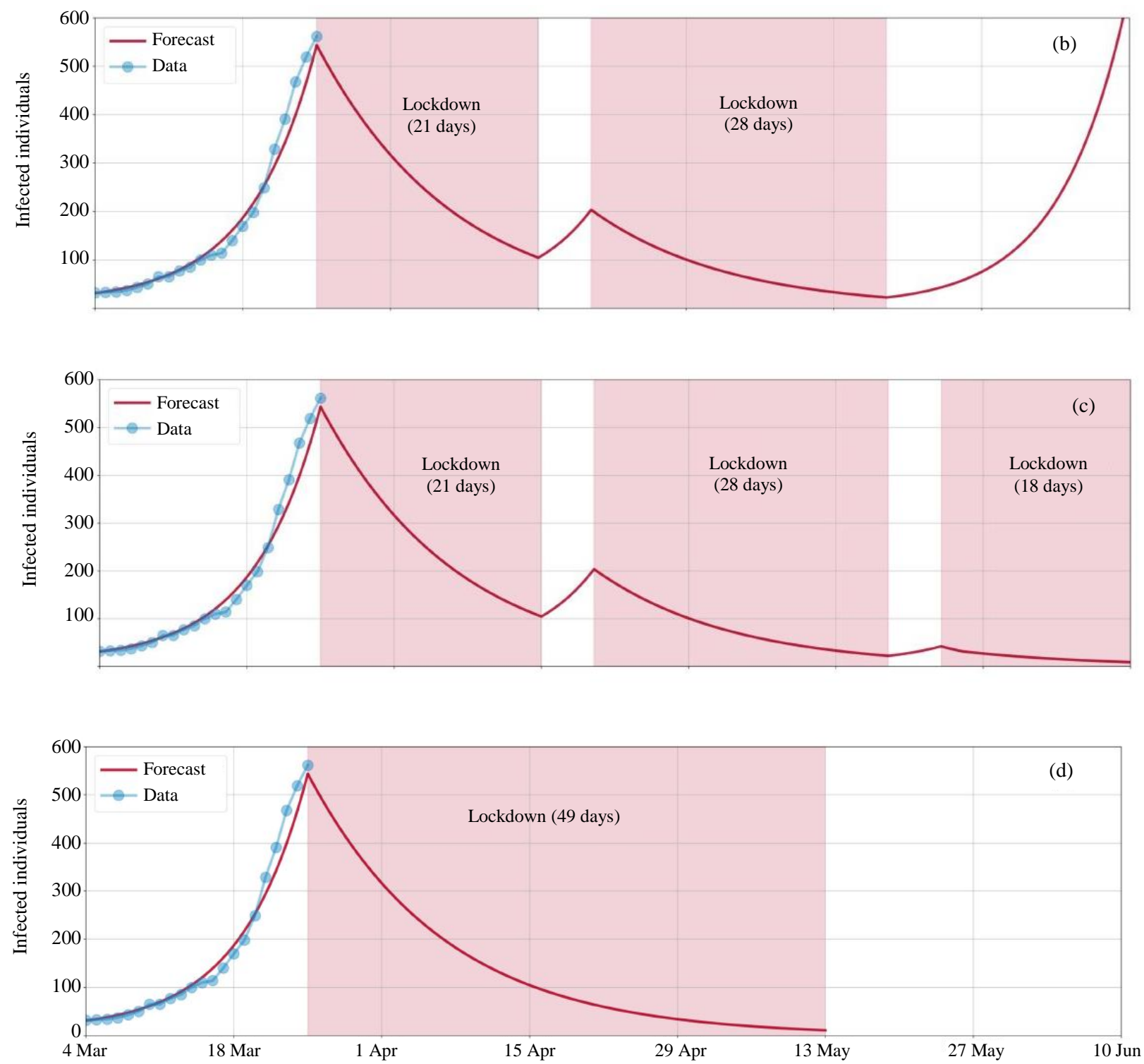

Fig. 2: The graphs of infected patients versus days as different schemes of series lockdowns (a), (b) and (c) and sustained lockdown (d) across the nation to restrict the chain of the novel COVID-19 pandemic in India using age-structured epidemiological Susceptible-Infected-Recovered (SIR) model (Reprinted with permission from (Singh and Adhikari, 2020). Copyright 2020 arXiv). *Note: Symptomatic cases were taken into consideration for the prediction of equilibrium stage of the pandemic. The inputs such as $\bar{\alpha}, \beta$ and $\gamma$ were taken as $1,0.0155$ and $1 / 7$, respectively

A 21 day lockdown (from 25th March, 2020 to 14th April, 2020) was found to be insufficient after careful investigation to ensure the equilibrium stage of the pandemic by the end of May, 2020 (Fig. 2). The outcome of the model was in favour of sustained lockdown with some relaxation for effective implementation of social distancing measures. It was found that the lockdown is imposed across the nation for a further 28 days after the relaxation period of 5 days from 25th March, 2020 and then the measure will be still insufficient to restrict the transmission chain of the SARS-CoV-2 (Fig. 2). As per the scheme, it was suggested that three successive lockdowns with a gap of 5 days as relaxation periods were required to bring the equilibrium stage of the pandemic (Fig. 2) (Appendix A) (Singh and Adhikari, 2020).

As per the guidance of the Honorable Prime Minister of India, Shri Narendra Modi, there was a voluntary public curfew of $14 \mathrm{~h}$ on 22nd March, 2020. Later on it was followed as mandatory and strict lockdowns were 
imposed in the major affected areas called as 'hotspots' in all major cities of the country. The nation further witnessed a strict 21 days nationwide lockdown from 24th March, 2020 as ordered by the Honorable Prime Minister of India. The nationwide lockdown was again extended up to 03rd May, 2020 by the Government of India on 14th April, 2020. This was later followed by another two-week extension with substantial relaxations. From 01st June, 2020, the government started "unlocking" the country (barring 'containment zones') in three unlock phases (Livemint, 2020; Singh and Adhikari, 2020; Inventiva, 2020a).

The factories and workplaces were shut down due to the nationwide lockdown which directly affected the livelihood of many migrant workers. Due to which, they were left with the only choice to turn back to their native villages. Thus, the Government of India asked the state government to set up relief camps for the migrant workers who are trying to return to their homes and issued orders for the protection of the rights of the migrant workers. It was admitted by the Supreme Court of India that the issues and problems of the migrant workers were still not completely solved. The Supreme Court ordered the state as well as the central government to provide free food, shelter and transport facilities to the migrant workers.
Most of the countries had lifted their lockdowns when the cases of novel COVID-19 started decreasing, but India has opted for a different plan. The WHO recommended the Government of India to test for SARS-CoV-2 for at least 14 days prior to lifting up the sustained lockdown to ensure positive rates were below the desired rate of $5 \%$. As per the report from the Johns Hopkins University's Coronavirus Resource Centre, the average rate of daily positivity for the novel COVID-19 in India was around 8-10\%. However, due to the unlock 1.0 on 07 th June, 2020, it was raised to $8.2 \%$ and on the next day it was almost $9.2 \%$. The increase in the positivity rate clearly throws light on the fact that the number of daily tests has been increased to unlock 1.0 in India. By May, 2020, India had done more than 10,00000 tests and the test positivity rate was just $3.8 \%$. In June, 2020, Central Government of India chose to lift the lockdown and announced that it is mandatory for every citizen to maintain social distancing in the market and all other social places. The physical distancing norms were also strictly followed during multiphased lockdown. Still, the novel COVID19 positive cases exponentially increased from a few hundred in March, 2020, to over 2,55000 when unlock 1.0 was declared in June, 2020 (Fig. 3) (Livemint, 2020; Singh and Adhikari, 2020; Inventiva, 2020a; 2020b; BBC News, 2020a; India Today, 2020a).

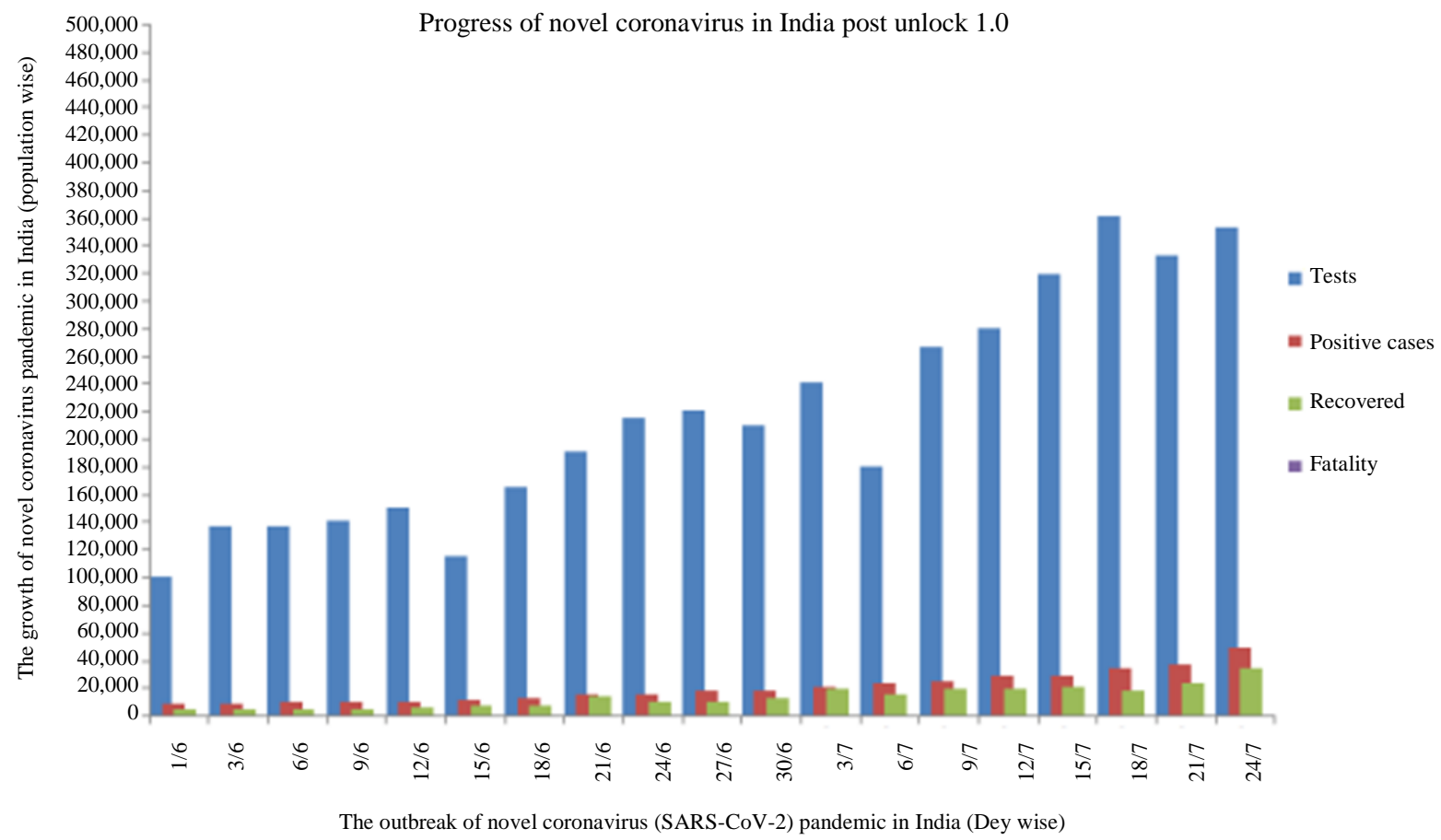

Fig. 3: The transmission of the novel Coronavirus Disease (COVID-19) post unlock 1.0 in India 
Mathematical models can forecast the spread of the novel COVID-19 pandemic and help inform public health interventions. The suitable input parameters for various scenarios such as partial lockdown, full lockdown, mass vaccination program, public education program, etc., are considered on the basis of (a) basic assumptions and (b) reported statistical data in published sources. In the present investigation, both stochastic and deterministic approaches are used to discuss the transmission of SARS-CoV-2 outbreak in India to fulfill the objectives. The stochastic model is used to estimate the probability of potential outcomes by having a random variation in one or more inputs over time in the proposed mathematical model. The stochastic models mainly depend on the variations in the risk of exposure to the disease and all other dynamic factors of the illness. In the case of the deterministic models, the individuals in the population are arranged in a different subgroups or small compartments, which directly represent a specific stage of the pandemic. Here, the letters $M, S, E, I$ and $R$ are used to represent variables such as different age groups of persons, susceptible, exposed, infected and recovered patients, respectively. Later on, the transition rates from one stage to another stage are mathematically expressed in the form of derivatives and thus the model is formulated using differential equations. Although when building mathematical models, it can be assumed that the size of the population in each of the compartments is differentiable with respect to time and that the pandemic process is deterministic (Gupta and Kanu, 2020; Atkins, 2010). The major goal of the present investigation is to study the transmission of the novel COVID-19 pandemic in India for various possible scenarios based on general assumptions and strategies inculcated through contact tracing using the values of contact ratios which are operated for the basic reproduction numbers, ' $R_{0}{ }^{\prime}$ ', with the aim to educate individuals to reduce the spread of the novel COVID-19 and vaccination program to assess their potential results in upcoming days. Furthermore, the transmission of the novel COVID-19 pandemic is also studied for majorly affected Indian cities on the basis of basic reproduction numbers (Atkins, 2010).

\section{Research Methodology}

Both deterministic and stochastic approaches are employed in the present mathematical investigations to study the transmissibility of SARS-CoV-2 pandemic in India. The Susceptible $(S)$-Infected $(I)$-Recovered $(R)$ i.e., SIR model is a compartmental model. When a Susceptible $(S)$ will come in an infectious contact with the infected individual, the susceptible will contract the disease and transition into the infected compartment. The symbol, ' $T$ represents the number of infectious individuals. These are individuals who have been infected and can infect the Susceptible $(S)$. The symbol, ' $R$ ' portrays the number of removed individuals. These are individuals who have been infected and have recovered from the disease or died.

The present investigation analyzes the spread of the novel COVID-19 pandemic in India and its majorly affected cities. The transmission of SARS-CoV-2 pandemic is due to the contact structure among the people and thus is governed by the parameter, ' $\beta$ (contact ratio). The parameter ' $\beta$ directly shows the result of the effectiveness of the social distancing. The transmissibility of the disease outbreak is studied and it is observed that the strictness with which social distancing in India is followed, is insufficient to break the chain of the novel COVID-19 (Atkins, 2010).

The SIR model considers the specific types of diseases which could cause an infected one to infect a susceptible after they encounter with each other. The variables $S, I$ and $R$, depict the population in every compartment for a particular instinct of time. The susceptible, infectious and removed individuals are a function of time and are represented by $S(t), I(t)$ and $R(t)$, respectively. The total number of individuals remains constant. The outbreak of such diseases in a specific population can be predicted by the mathematical model. The dynamics of the transition between these compartments can be modelled with the help of some parameters. These are ' $\beta$ ' and can be referred as transmission rates. The parameter, ' $\gamma$ ' is the reciprocal of the infectious period, which is the time duration in which the infected can transmit the disease (Gupta and Kanu, 2020; Atkins, 2010; Mazumder et al., 2020).

The distribution of population within these compartments at a given time, $t$, using the general SIR model are shown using a set of ordinary differential Equations (1) to discuss the transmission of the novel COVID-19 pandemic in India:

$$
\left.\begin{array}{l}
\begin{array}{l}
S+I+R=N \\
\frac{d S}{d T}=-\frac{\beta I S}{N}
\end{array} \\
\frac{d I}{d T}=\frac{p \beta I S}{N}-I(\gamma+\mu) ; \\
\frac{d R}{d T}=\gamma(I)
\end{array}\right\}
$$

\section{The Modified $S I_{S} I_{N} R$ Transmission Network Model for Modeling and Simulation of the Novel COVID- 19 Outbreak in India}

The $S I_{S} I_{N} R$ transmission network model can be regarded as a derivative of the basic SIR model (Fig. 4). In the $S I_{S} I_{N} R$ model, we include the asymptomatic compartment which turns the $S I R$ model into the $S I_{S} I_{N} R$ model (Fig. 5a). The modified $S I_{S} I_{N} R$ transmission network model is partitioned into classes such as susceptible $S(t)$, symptomatic infected $I_{S}(t)$, asymptomatic infected $I_{N}(t)$ and removed $R(t)$; where $N+I_{S}+I_{N}+R=N$. 


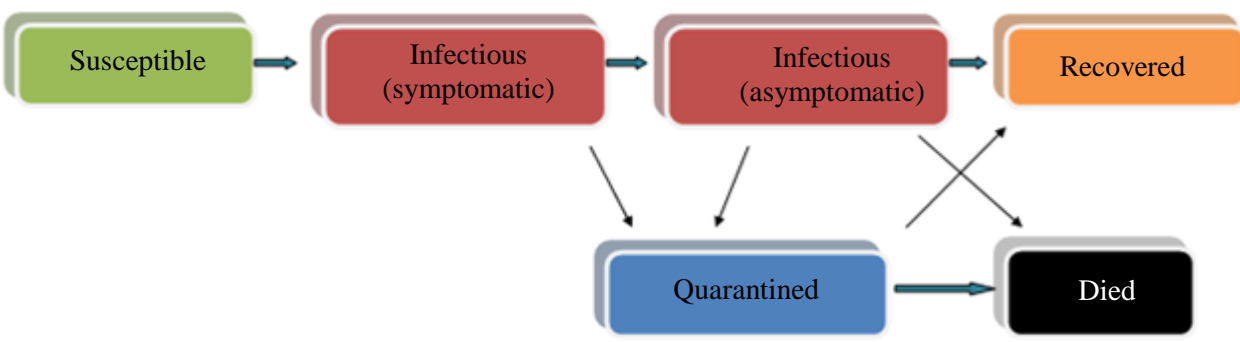

Fig. 4: The schematic representation of modeling of the novel Coronavirus (COVID-19) pandemic using $S I_{N} I_{N} R$ model in India

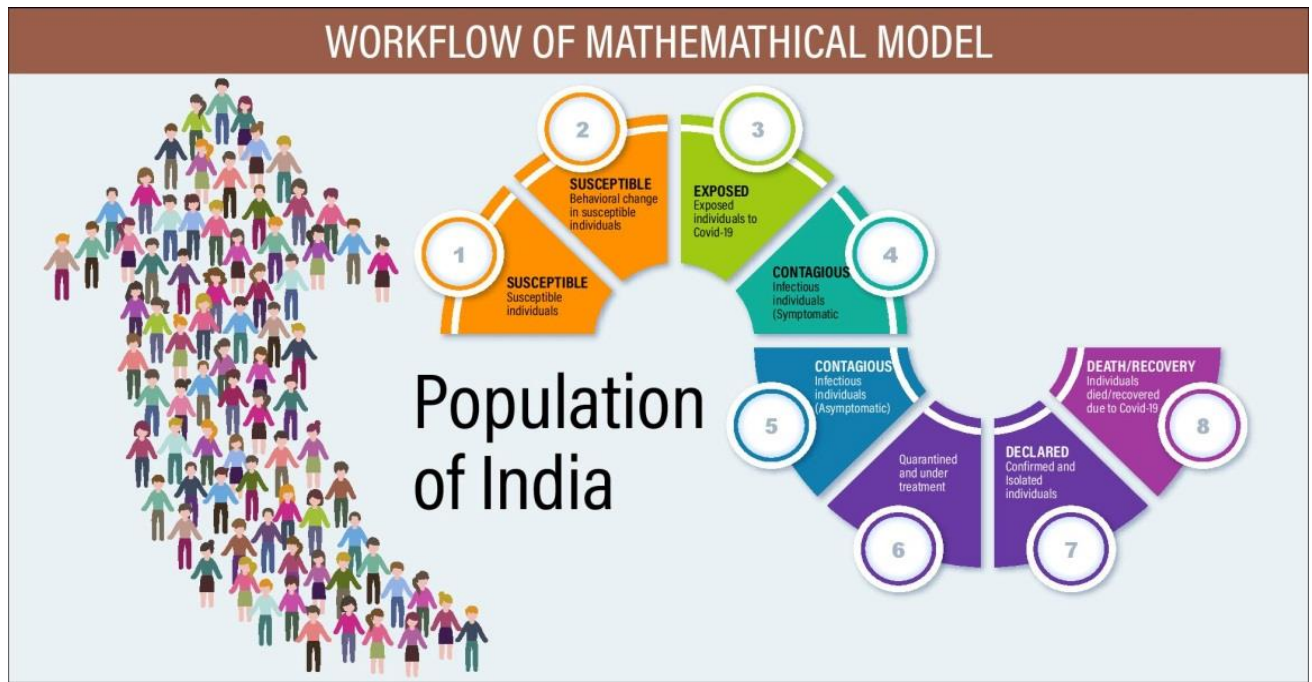

(a)

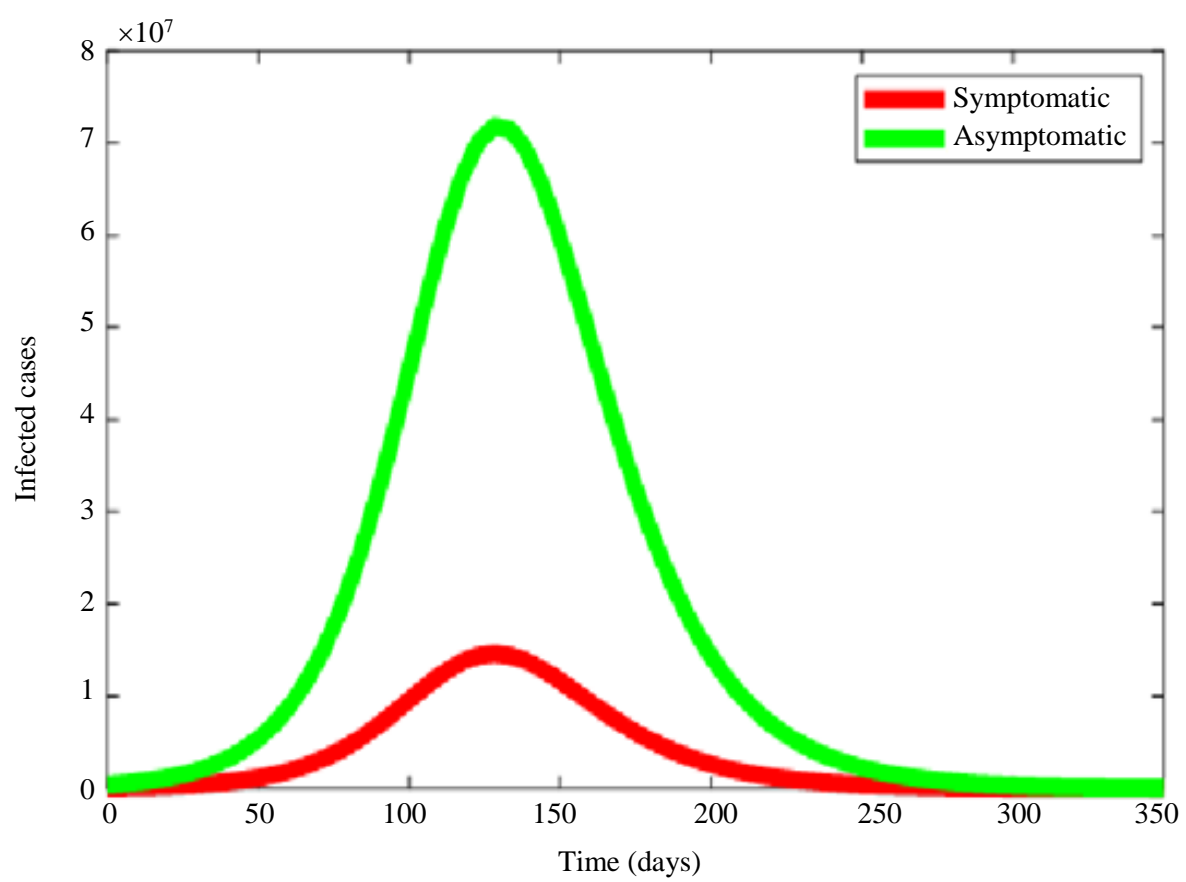

(b)

Fig. 5: The outbreak of SARS-CoV-2 pandemic in India. (a) The illustration of mathematical model of SARS-CoV-2 pandemic in India. (b) The transmission of the novel COVID-19 pandemic is shown with the graph of infected patients (both symptomatic and asymptomatic) versus time (days) using deterministic approach of $S I_{S} I_{N} R$ transmission network model 
Those contracting the disease entered into the infected classes at a rate of ' $p$ ' i.e., the symptomatic class, or ' $(1-p)$ ' entered into the asymptomatic class. Here, the infected individuals gets recovered from the infection and entered into the removed compartment at a rate of ' $\gamma$ ' or may die due to the illness at a rate of ' $\mu$ '. Those who are within the asymptomatic compartment exit into the removed category at the rate of ' $\gamma$ ' (Fig. 5b) (Gupta and Kanu, 2020; Atkins, 2010; Mazumder et al., 2020; Times Network, 2020; Pai et al., 2020).

Those contracting the disease entered into the infected classes at a rate of ' $p$ ' i.e., the symptomatic class, or '(1-p)' entered into the asymptomatic class.

The distribution of population within these compartments at a given time, $t$, using the modified $S I_{S} I_{N} R$ transmission network model are shown using the set of ordinary differential Equations (2) to discuss the transmission of the novel COVID-19 pandemic in India:

$$
\left.\begin{array}{l}
S+I_{S}+I_{N}+R=N \\
\frac{d S}{d T}=-\frac{\beta\left(I_{S}+I_{N}\right) S}{N} \\
\frac{d I}{d T}=\frac{p \beta I S(I+I)}{N}-I(\gamma+\mu) \\
\frac{d I_{A}}{d T}=\frac{(1-p) \beta S\left(I_{S}+I_{A}\right)}{N}-\gamma I_{A} \\
\frac{d R}{d T}=\gamma(I+I)
\end{array}\right\}
$$

The basic reproduction number, ' $R_{0}$ ', or basic reproductive number, or basic reproductive rate of an infection can be thought as the expected number of cases directly generated by one case in a population where all individuals are susceptible to infection. Here, the basic reproduction number, ' $R_{0}$ ' is calculated using the formula, $R_{0}=\beta /(\gamma+\mu+\alpha)$, (where, ' $\alpha$ ' represents persons who entered into the removed class and have resistance to fight against SARS-CoV-2 for a certain time, afterwards they will be considered a susceptible class) (Atkins, 2010; Tiwari, 2020; Rajesh et al., 2020).

\section{The Outbreak of the Novel COVID-19 Pandemic in India}

According to the report of the Hindustan Times, around $75 \%$ of the total novel COVID-19 cases in Punjab, 50\% in Karnataka, 65\% in Maharashtra and $75 \%$ in Uttar Pradesh were asymptomatic. Nearly $82 \%$ of the novel COVID-19 patients in Assam (reported at the time) were asymptomatic. On 20th April, 2020, the health ministry warned that $80 \%$ of all coronavirus patients were asymptomatic or showing mild symptoms. Around $80 \%$ are asymptomatic or show mild symptoms. Around $15 \%$ patients turned into severe cases and $5 \%$ cases turned into critical cases (India Today, 2020a; Mackolil and Mahanthesh, 2020; Bhola et al., 2020). As ' $p$ ' represents the symptomatic class and '(1-p)' represents the asymptomatic class, therefore, we consider $p=0.2$ as $80 \%$ cases are asymptomatic).

There is a period between which a person gets infected and is confirmed positive. Hence, the total number of infected people is greater than the total number of confirmed positives in an exponential growth phase and the total number of infected people is close to the total number of confirmed cases when the growth is halted. This is the reason many developed countries failed to halt the growth of the virus in the exponential phase. The incubation period for the novel COVID-19, which is the time between exposure to the virus (becoming infected) and symptom onset, is on average 5-6 days, however can be up to 14 days. Although the overall fatality rate is estimated to range from $2-3 \%$, the disease can be fatal to elderly people (about 27\%) whose age is more than 60 and those with an underlying medical history (Gupta and Kanu, 2020; Atkins, 2010). We assumed that the recovery rate ' $\gamma$ ' would remain constant for the Indian population around 0.1 .

In Table 1 , the contact ratio, ' $\beta$ ' is varied to depict the outbreak of novel COVID-19 as per the cases explored in the literature, so far for the outbreak of the novel of the COVID-19 pandemic in India (Atkins, 2010; Rihan and Anwar, 2012; Daughton et al., 2017; Chatterjee et al., 2020; Dhama et al., 2020). The recovery rate, ' $\gamma$ ' is taken constant which is around 0.1 . It can be calculated by the formula ( 1 /infectious period), the average death rate, ' $\mu$ ' is taken as 0.025 . The parameter ' $p$ ' depicts the ratio of symptomatic infected to total infected and as $80 \%$ patients are asymptomatic, thus ' $p$ ' is taken as 0.2 .

\section{Stochastic Model}

The model presented here is modified with the inclusion of demographic stochasticity, which is defined as "fluctuations in population processes that arise from the random nature of events at the level of the individual". Although the probability of each event here is fixed, there is change in the outcomes because of the chance of individuals to get in contact with the infected ones. A deterministic model is the one which yields the same results under the same initial conditions. Stochastic models, on the other hand, include an element of randomness indicative of the real world. The main outcome of the analysis was to study the extinct of SARS-CoV-2 pandemic with the help of the stochastic model regardless of the value of the basic reproduction number $\left(R_{0}\right)$. In the case of a very long time to extinction, the conclusion was that there may be the existence of a quasi-stationary probability distribution whose mean almost agrees with the deterministic pandemic equilibrium (when $R_{0}>1$ ). 
Table 1: Inputs in the present investigation for the modeling of the novel COVID-19 pandemic in India

\begin{tabular}{lll}
\hline Symbols & Parameters & Values \\
\hline$\beta$ & Contact ratio & Varied \\
$\gamma$ & Average recovery rate & 0.100 \\
$\mu$ & Average death rate due to the novel COVID-19 & 0.025 \\
$\rho$ & Ratio of symptomatic patients to total patients & 0.200 \\
\hline
\end{tabular}

Table 2: Data of most affected cities in India

\begin{tabular}{llllrl}
\hline City & Population & Active cases up to 28th July, 2020 & $\beta$ & $\gamma$ & $\mu$ \\
\hline Delhi & $3,02,91,000$ & 10,887 & Varies & 0.1 & 0.025 \\
Pune & $66,39,347$ & 48,680 & Varies & 0.1 & 0.025 \\
Mumbai & $2,04,11,000$ & 21,812 & Varies & 0.1 & 0.025 \\
Chennai & $1,09,7,1108$ & $13,064 \mathrm{a}$ & Varies & 0.1 & 0.025 \\
Ahmedabad & $56,00,000$ & 3,343 & Varies & 0.1 & 0.025 \\
\hline
\end{tabular}

Using the modified $S I_{S} I_{N} R$ transmission network model, we are considering five different events that can occur, each causing the numbers in the relative classes to increase or decrease by one (Gupta and Kanu, 2020; Atkins, 2010; Rihan and Anwar, 2012; Daughton et al., 2017; Chatterjee et al., 2020):

- $\quad$ Transmission occurs at rate $\beta \operatorname{Sp}\left(I_{S}+I_{S}\right) / N$; Result $S$ $\rightarrow S$-1 and $I \rightarrow I+1$

- Transmission occurs at rate $\beta S(1-p)(I+I) / N$; Result $S \rightarrow S-1$ and $I \rightarrow I+1$

- $\quad$ Recovery occurs at rate $\gamma I_{S}$; Result $I_{S} \rightarrow I_{S^{-}} 1$ and $R$ $\rightarrow R+1$

- $\quad$ Death occurs at rate $\mu I_{S}$; Result $I \rightarrow I-1$

- $\quad$ Recovery occurs at rate $\gamma I$; Results $I_{N} \rightarrow I_{N^{-}} 1$ and $R$ $\rightarrow R+1$

As the transmission occurs at the rate $\beta \operatorname{Sp}\left(I_{S}+I_{N}\right) / N$, initially, which results into $S \rightarrow S-1$ and $I \rightarrow I+1$, one person gets removed from the group of susceptible and gets added to the group of infected ones. The same process is repeated when the transmissions occur at the rate $\beta S(1-p)(I+I) / N$. In case of the recovery rate $\gamma I_{S}$ (symptomatic as well as asymptomatic), one person is reduced from the group of infected group and gets added to the group of recovered ones. In case of death rate $\mu I_{S}$, one person gets reduced from the group of individuals of infected class and dies (Atkins, 2010). The following data were used as inputs to model the outbreak of the novel COVID-19 pandemic in various cities in India, stochastically.

Table 2 depicts the population and active cases of the most affected cities in India which includes Delhi, Pune, Mumbai, Chennai and Ahmedabad. Here, the value of contact ratio ' $\beta$ ' is varied to predict the outbreak of novel COVID-19 under different scenarios. We have plotted the outbreak of SARS-CoV-2 pandemic for the range of values of the basic reproduction number, ' $R_{0}$ ', from 1.25 , $1.5,2.0,2.5$ and 3.0 based on the values of contact ratio ' $\beta$ ' (Atkins, 2010; Rihan and Anwar, 2012; Dhama et al.,
2020; Mandal et al., 2020) using the modified $S I_{S} I_{N} R$ transmission network model to study the outbreak of the pandemic in-depth. The average recovery rate ' $\gamma$ ' is calculated to be 0.1 (1/infectious period). The average death rate ' $\mu$ ' is taken as 0.025 .

\section{Validation of the Present Stochastic and Deterministic Models based on Modified $S I_{S} I_{N} R$ Transmission Network Model with Datasets of Meningitis Epidemic Outbreak in University of Central Florida (UCF) Campus}

Table 3 represents the values for the parameters which were used to plot the validation graphs. It contains the values of variables which include probability of transmission of infection, vaccination trial, contact rate and reproduction rate. The values of probability of transmission $(q)$ of SARS-CoV-2 pandemic for four different models such as base, education, vaccination and education and vaccination models (Appendix B) are discussed herewith as per the meningitis outbreak case study (Atkins, 2010) to prove the efficacy of the present stochastic and deterministic MATLAB programs based on the modified $S I_{S} I_{N} R$ transmission network model. As people get knowledge and awareness about protecting themselves from the infection, the contact ratio as well as the reproduction rate goes on reducing (Atkins, 2010).

\section{Validation of the Stochastic Model}

For small populations, a stochastic model may be more appropriate. In such a case, the stochastic model, which is concerned with mimicking the random or probabilistic event, would be more suitable.

In the stochastic model (Fig. 6), there are three curves which include: Number of susceptible (blue), number of infected (red) and number of recovered (yellow) plotted against the time (days). The curve for the susceptible cases here showed the number of susceptible around 50,000, which is the population of the University of Central 
Florida campus (Atkins, 2010). The curve here depicts that the number of susceptible will reach to zero in a week. The second curve plotted (red) shows the number of infected ones. It depicts the number of infected cases which starts increasing from the fourth day of the spread of meningitis infection with probably 2,000 people getting infected. The number will rise till the sixth day with near about 4,800 people getting infected. Till the end of the second week of spread of infection, the number of people getting infected will start reducing and move towards the normal condition. The last curve (yellow) here shows the number of people in the campus who were recovered from the infection. The recovery of the infected started from the first week of infection spread. Till the end of the first week, almost 2,000 people got recovered from the infection. Later on, by the end of the second week, almost 4,000 people were recovered. By the end of month more than 5,000 people were recovered (Gupta and Kanu, 2020; Atkins, 2010). The present stochastic MATLAB programs based on the modified $S I_{S} I_{N} R$ transmission network model are validated well under all three cases using the similar inputs for the meningitis outbreak case.

\section{Validation of the Deterministic Model}

In a deterministic model, the same results are observed for the same initial conditions every time. On the other hand, some randomness is always included in the stochastic model which depicts the real world situation. In Figs. 7-10, the deterministic curves represent the number of infected people versus the time (days). The blue-coloured curves plotted by (Atkins, 2010), are superimposed by the pink-coloured curves using the present deterministic MATLAB program based on the modified $S I_{S} I_{N} R$ transmission network model.

\section{Validation of the base Model}

Here, the graph shows that if the infection continues to spread without any education or vaccination program, then by the end of the first week almost 4,000 people would be infected in a rapid manner. Later on, the number of infected persons will go on reducing almost after a week with 500 infected cases. Furthermore, by the end of the month, the situation in the university will be back to normal (Atkins, 2010).

\section{Validation of the Education Model}

In the model, the infection will start rising from the end of the first week with almost 200 people getting infected. The model depicts that if people were made aware about the spread of infection in the campus of university from the beginning itself, the overall growth of the infection could be definitely lowered.
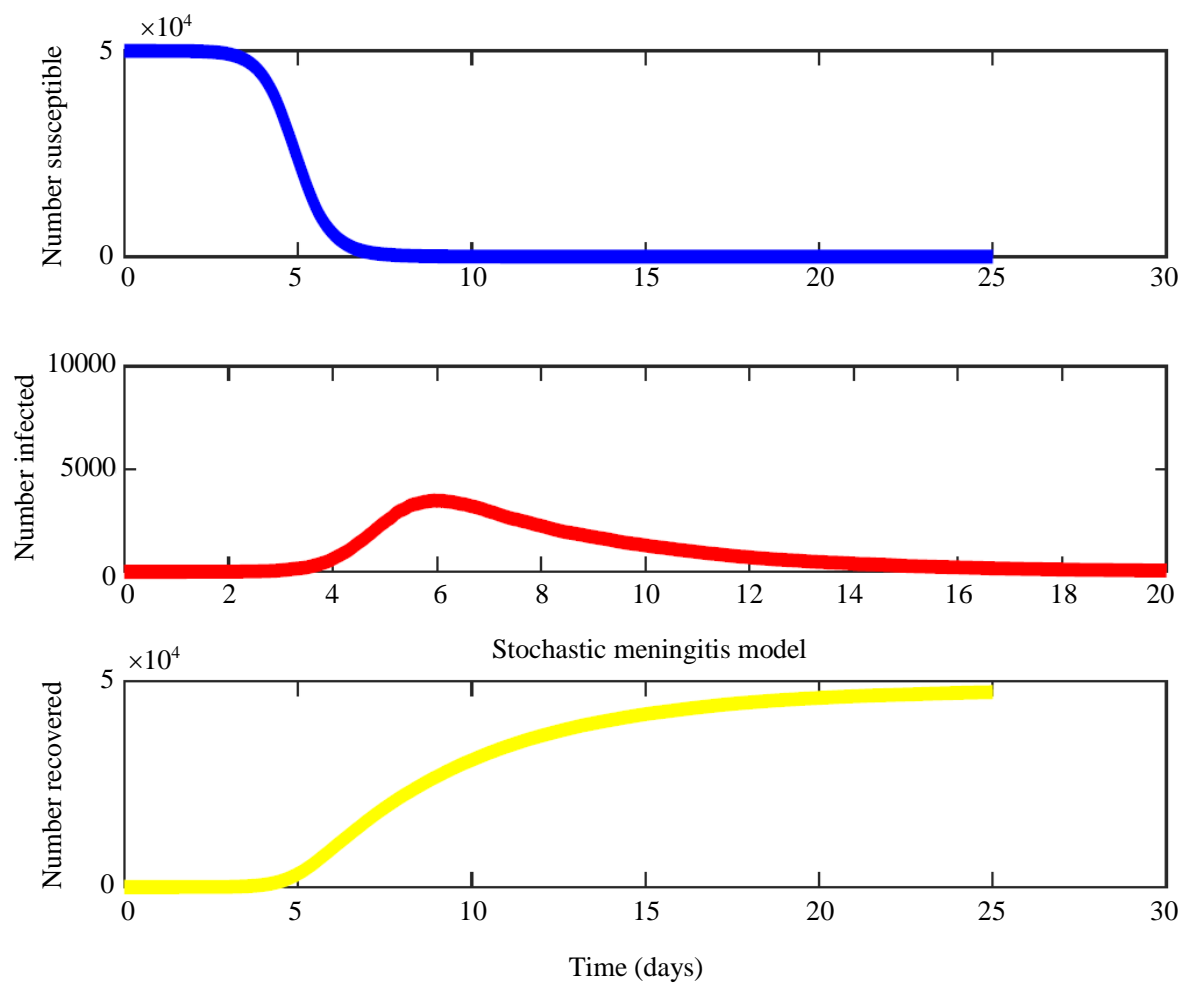

Fig. 6: Stochastic model for meningitis outbreak in University of Central Florida (UCF) campus 


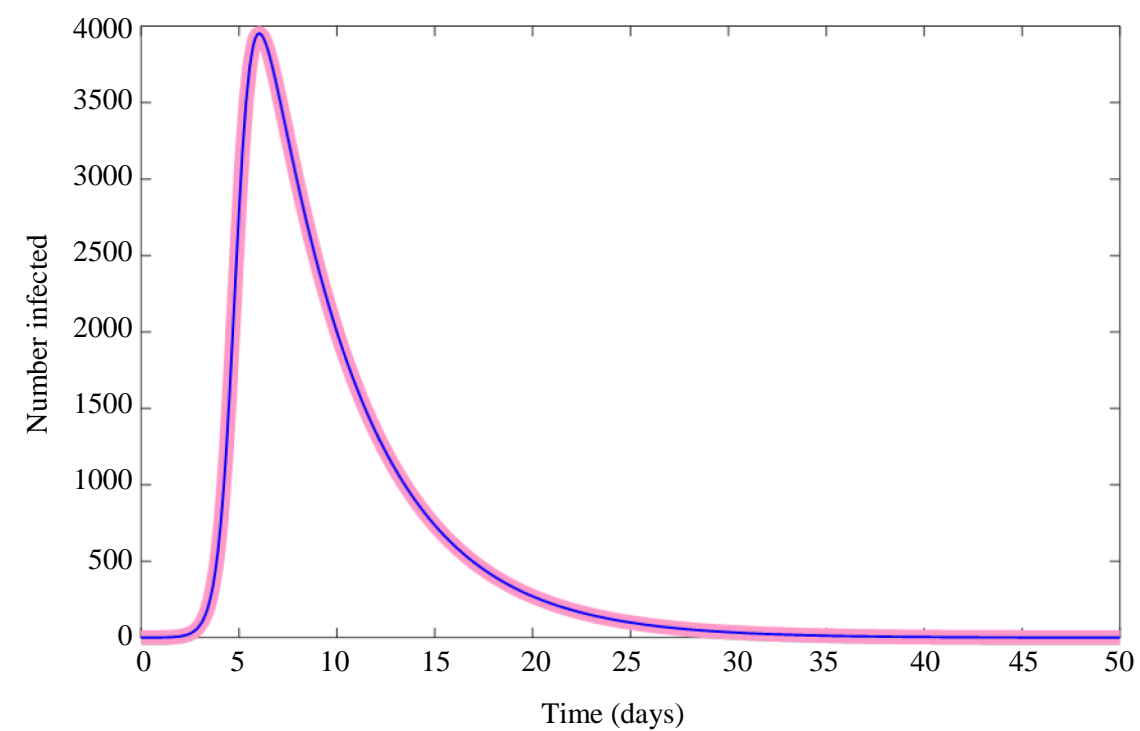

Fig. 7: Deterministic (base) model for meningitis outbreak in University of Central Florida (UCF) campus

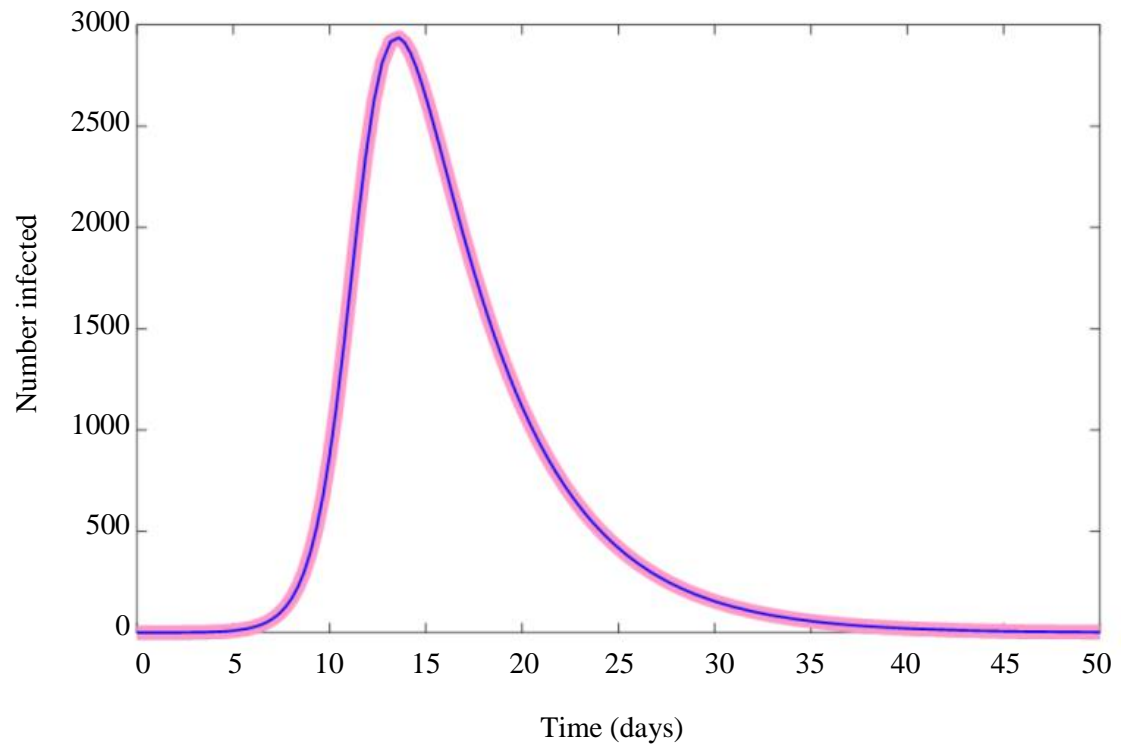

Fig. 8: Deterministic (education) model for meningitis outbreak in University of Central Florida (UCF) campus

Table 3: Parameters for the study of an outbreak of meningitis epidemic in University of Central Florida (UCF) campus

\begin{tabular}{lllcll}
\hline Parameters & Variable & $\begin{array}{l}\text { Base } \\
\text { model }\end{array}$ & $\begin{array}{l}\text { Education } \\
\text { model }\end{array}$ & $\begin{array}{l}\text { Vaccination } \\
\text { model }\end{array}$ & $\begin{array}{l}\text { Education and } \\
\text { Vaccination model }\end{array}$ \\
\hline Probability of transmission of infection & $q$ & 0.10 & 0.05 & 0.1 & 0.05 \\
Number of peoples vaccinated & $v_{0}$ & 0.00 & 10.00 & $51 \%$ & $61 \%$ \\
Calculated contact ratio & $\beta$ & 2.34 & 1.05 & 1.15 & 0.46 \\
Basic reproduction rate & $R_{0}$ & 7.80 & 3.50 & 3.8 & 1.5 \\
\hline
\end{tabular}

As shown by the curve till the end of the second week, the maximum number of people will get infected. Almost 3,000 people will get infected by the end of the second week. Later on, the number of infected people will go on decreasing due to awareness about the prevention of the infectious disease. By the end of month the number of infected people will be lowered and the situation will be back to normal (Atkins, 2010). 


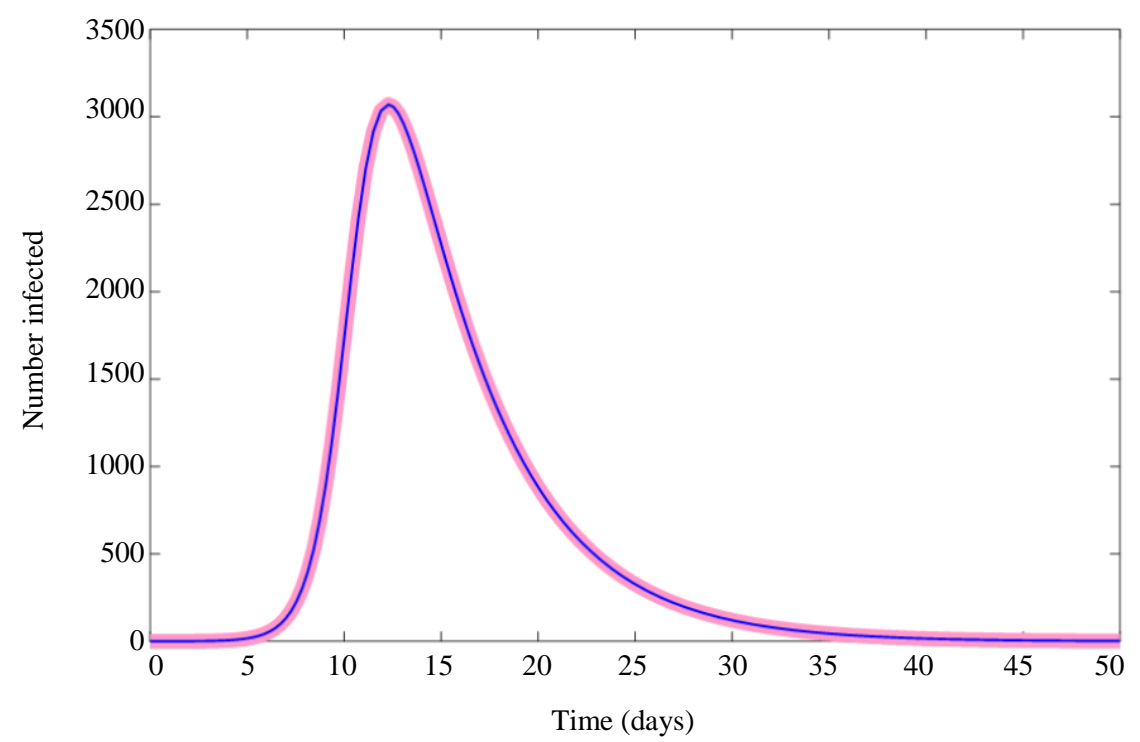

Fig. 9: Deterministic (vaccination) model for meningitis outbreak in University of Central Florida (UCF) campus

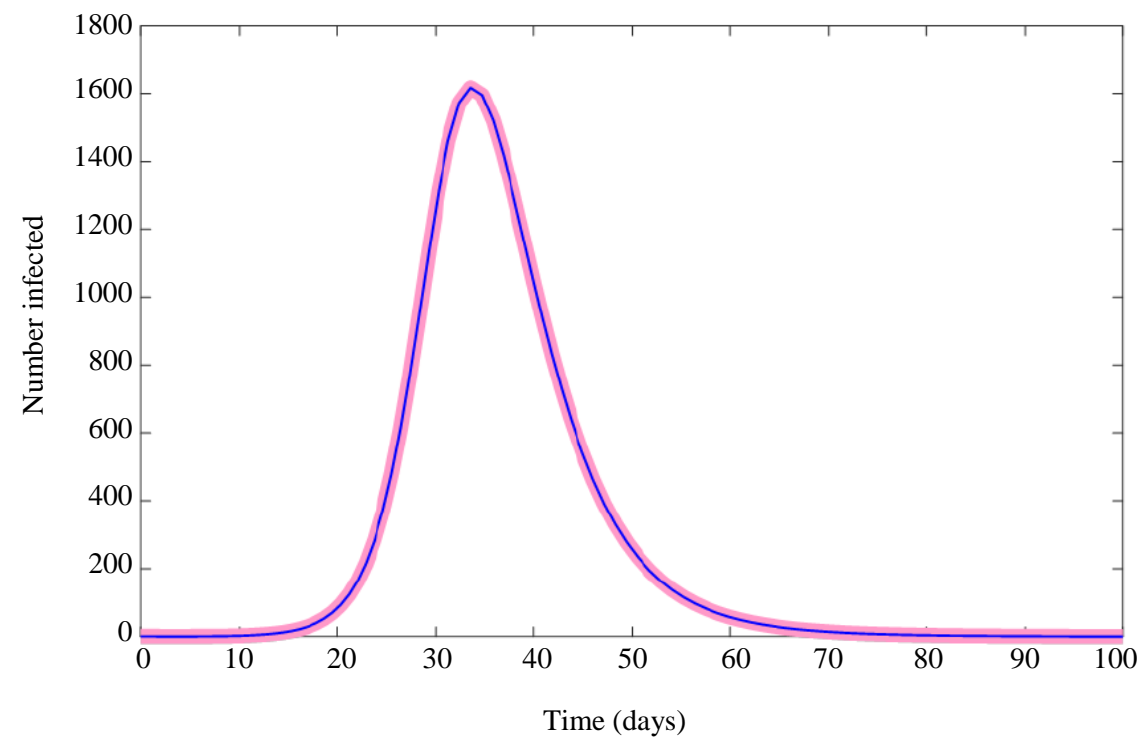

Fig. 10: Deterministic (education and vaccination) model for meningitis outbreak in University of Central Florida (UCF) campus

\section{Validation of the Vaccination Model}

The model depicted that the number of infected people will start rising by the end of the first week of infection with almost 100 cases.

As shown in Table 3, there would be $51 \%$ of people vaccinated, which resulted in the lower number of cases, i.e., around 3,000 (maximum) cases of infection by the second week. The number of people getting infected will start reducing by the second week of infection. By the end of the month, this situation will turn out to be normal (Atkins, 2010).

\section{Validation of the Education and Vaccination Model}

The model shows the result of the combined education and vaccination model. As shown in Table 3 , there would be around $61 \%$ of people who will be aware about the preventive measures and would be vaccinated as well.

This will ultimately lower the number of maximum people getting infected and then it would be around 600 (maximum) cases of infection after almost 40 days. The rise in the number of infected people in this model will start late as compared to other models. By the third week, there would be almost 50 people getting infected. 
After almost 40 days, the number of infected people will start lowering. Later on, after 3 months, the situation may be back to normal (Atkins, 2010).

\section{Results}

Deterministic Susceptible (S)-Infected (Symptomatic) (IS) Infected (Asymptomatic) (I $I_{N}$ ) Recovered (R) i.e., $S I_{S} I_{N} R$ Models for Modeling and Simulation of the Outbreak of Novel Coronavirus Diseases (COVID19) in India

In the present investigation, the basic reproduction number, ' $R_{0}$ ', are calculated using different values of contact rates, $\beta$, based on the novel deterministic susceptible $(S)$-infected (symptomatic) $\left(I_{S}\right)$ infected (asymptomatic) $\left(I_{N}\right)$ recovered $(R)$ approach to discuss the transmission of the novel COVID-19 outbreak. A suitable computer routine is developed in MATLAB based on Ordinary Differential Equations (ODEs) in conjunction with Runge-Kutta method with a variable time step for efficient simulation of the growth of SARSCoV-2 under control and prevention strategy program in India. The deterministic model is further classified into three submodels such as (a) base model, $q=0.1$, (b) education model, $q=0.05$, (c) education and vaccination model $q=0.1$ for in-depth analysis of the outbreak of the pandemic (Atkins, 2010; Rihan and Anwar, 2012; Dhama et al., 2020; Mandal et al., 2020).

The spread of the novel COVID-19 pandemic is plotted for entire nation (Fig. 11). The graph, infected cases versus time (days) is plotted for SARS-CoV-2 outbreak to predict its behavior in due course of time. The mitigatory measures such as strict lockdown and partial lockdown across the nation are responsible to restrict the spread of pandemic. According to the graph the pandemic is approximately expected to end after 300 days from present day, i.e., 28th July, 2020. In Fig. 11, the ' $R_{0}$ ' is calculated around 1.25 following the deterministic strategies for entire India. The symptomatic cases are found to be around $80 \%$ less than asymptomatic cases (Atkins, 2010; Rihan and Anwar, 2012; Mandal et al., 2020). The suitable graph has been plotted on the basis of values of ' $R_{0}$ ' using deterministic mathematical models based on the modified $S I_{S} I_{N} R$ transmission network model. The peaks of infected cases (both symptomatic and asymptomatic cases) as well as end of SARS-CoV-2 pandemic are estimated soon after 31st July, 2020 in the month of December, 2020 (Fig. 11) and by the end of March, 2021, respectively.

\section{Impact of Community Transmission Phase of SARS- $\mathrm{CoV}-2$ in India}

As per the joint statement by Indian medical professional associations, nonuniform and rational strategies of lockdown across the nation such as movement of migrant workers, social gathering unlock 1.0 and 2.0, etc., resulted in community spread of the novel COVID-19 disease. India must be well equipped to counter the probability of community transmission of the COVID-19. Easing of lockdown will lead to more widespread and rapid transmission of coronavirus. Some of the experts suggested that India has already entered the community (Gupta and Kanu, 2020) transmission through the stage III of the novel COVID-19. As if one looks at the spread of people without a history of travel or history of contact, certainly there are several such cases.

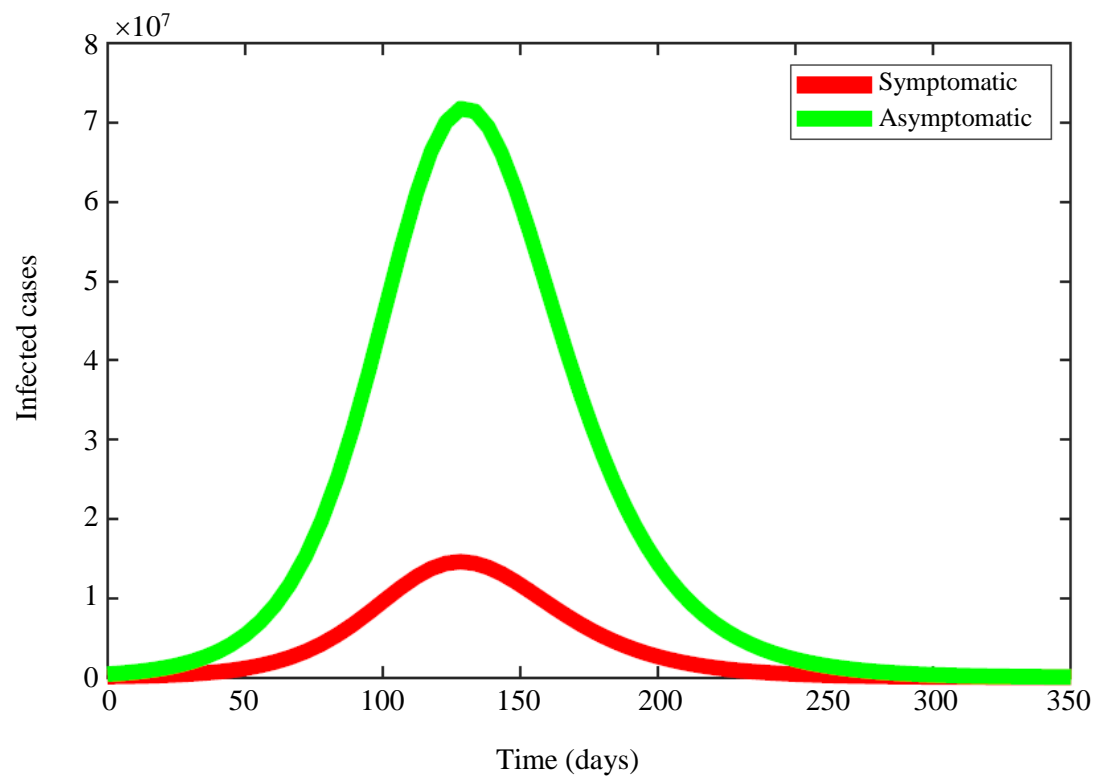

Fig. 11: Graphical simulation of the novel COVID-19 outbreak. Base model yields $R_{0}=1.25$ using $\beta=0.15625$ 


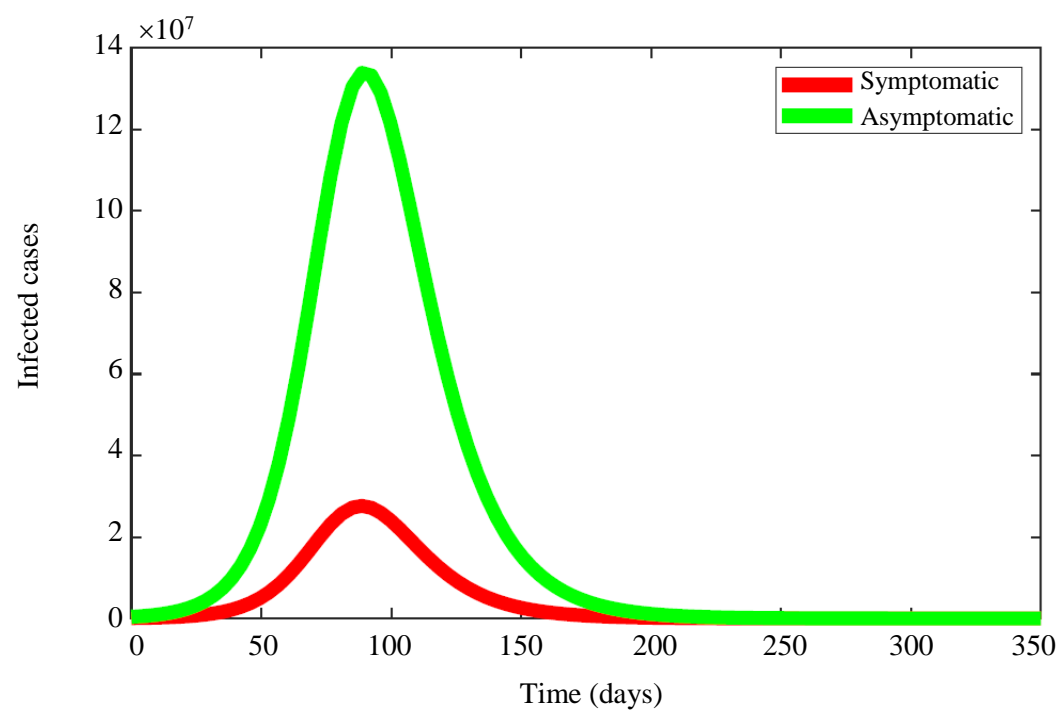

Fig. 12: Graphical simulation of the novel COVID-19 outbreak. Base model yields $R_{0}=1.5$ using $\beta=0.1875$

Furthermore, governmental action and some misuse of mitigatory measures have influenced the spread of the pandemic (Fig. 12). As per the data reported in some published sources; the pandemic is modeled with the basic reproduction number, ' $R_{0}$ ', to validate the novel deterministic modeling approach of SARS-CoV-2 pandemic. The pandemic is predicted to end after 250 days from the present day, i.e., 28th July, 2020, using the current value of $\beta=0.1875$. In Fig. 12 , the ' $R_{0}$ ' is calculated around 1.5 following the deterministic strategies for the entire India (Atkins, 2010; Rihan and Anwar, 2012; Chang et al., 2020).

Social distancing is the only proven solution for halting corona virus growth as of now. Nation practices strict social distancing in the lockdown, but still due to several factors, we see failures in some lockdowns. Here ' $\beta$ ' is the transmission rate and ' $\gamma$ ' is the average recovery rate. With quarantine and isolation, the increasing number of infected slowly became constant. In the context of India, this model may be useful as a rigorous social distancing measure has been introduced and hence does not consider factors like age, etc. The parameter, ' $\beta$ ' governs the transmission efficiency of the virus in society, since lockdowns and social distancing measures focus on reducing the transmission, reduction in ' $\beta$ ' in different stages will reflect how better the lockdown was performed. The mitigatory measures imposed by Indian government have influenced the spread of pandemic (Fig. 13). With the further change in parameters such as contact rate, ' $\beta$ ' reported in some published sources, the pandemic is modeled for the basic reproduction number, ' $R_{0}$ ', to throw light on the novel deterministic modeling approach of SARS-CoV-2 pandemic. The pandemic is predicted to end after 200 days from the present day, i.e., 28th July, 2020, using the current value of $\beta=0.25$. In Fig. 13 , the ' $R_{0}{ }^{\prime}$ is calculated around 2.0 following the deterministic strategies for the entire nation (Atkins, 2010; Rihan and Anwar, 2012; Sahasranaman and Kumar, 2020; Dhanwant and Ramanathan, 2020).

The testing of novel COVID-19 is essential in the direction of containing the spread of virus. The delays in testing may seriously reduce the ability of the population to protect it. The testing for the novel COVID-19 virus in India was inadequately conducted in the beginning, which might have not generated sufficient data on the number of people affected. Initially, only persons with a travel history to high risk countries were tested. Therefore, this case can be varied with different values of contact rate, $\beta$, as reported in some published sources; the pandemic is modeled for the basic reproduction number, ' $R_{0}$ ', to observe the transmission of the novel COVID-19 pandemic using the novel deterministic approach to prove the efficacy of the present model (Fig. 14).

The pandemic is predicted to end after 150 days from the present day, i.e., 28th July, 2020, using the current value of $\beta=0.3125$. In Fig. 14 , the ' $R_{0}$ ' is calculated around 2.5 following the deterministic strategies for the entire India (Atkins, 2010; India Today. 2020a).

As of 14th March, 2020, the National Institute of Virology, Pune, has tested around 5,900 samples from individuals across the country, which was quite low compared to other countries. It was also felt that community transmission may go undetected without adequate testing. Testing for community transmission began on 15th March, 2020. The Department of Health Research and the Indian Council of Medical Research (DHR-ICMR) have started testing random samples of people who exhibit flu-like symptoms and samples from 
patients without any travel history or contact with infected persons. Later on, it was also decided to include all pneumonia cases, regardless of travel or contact history after the country saw a sharp increase in the number of cases. The ICMR further revised the testing strategy and allowed testing of people showing symptoms for a week in the hotspot areas of the country, regardless of travel history or local contact to the patient. Total 25,12,388 samples were tested by 20th May, 2020, out of which 1,06,750 have been confirmed positive. Finally, these are discussed with the contact rate, ' $\beta$ ' as reported in some published sources; the pandemic is modeled for the basic reproduction number, ' $R_{0}$ ', to observe the transmission of the novel COVID-19 pandemic using the novel deterministic approach to prove its efficacy (Fig. 15). The pandemic is estimated to reach its end after 100 days from the present day, i.e., 28 th July, 2020, using the current value of $\beta=0.375$. In Fig. 15 , the ' $R_{0}$ ' is calculated around 3.0 following the deterministic strategies for the entire India (India Today, 2020a; Atkins, 2010; Rihan and Anwar, 2012; Ramola, 2020; The Times of India, 2020a).

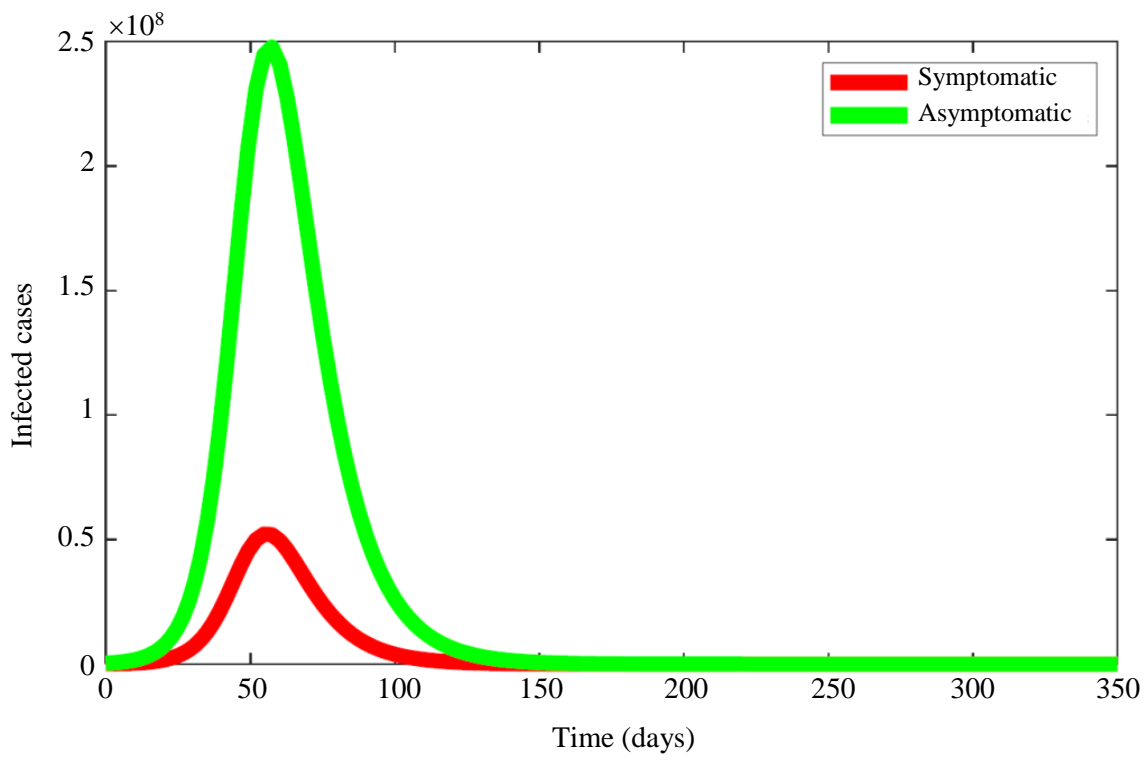

Fig. 13: Graphical simulation of the novel COVID-19 outbreak. Base model yields $R_{0}=2.0$ using $\beta=0.25$

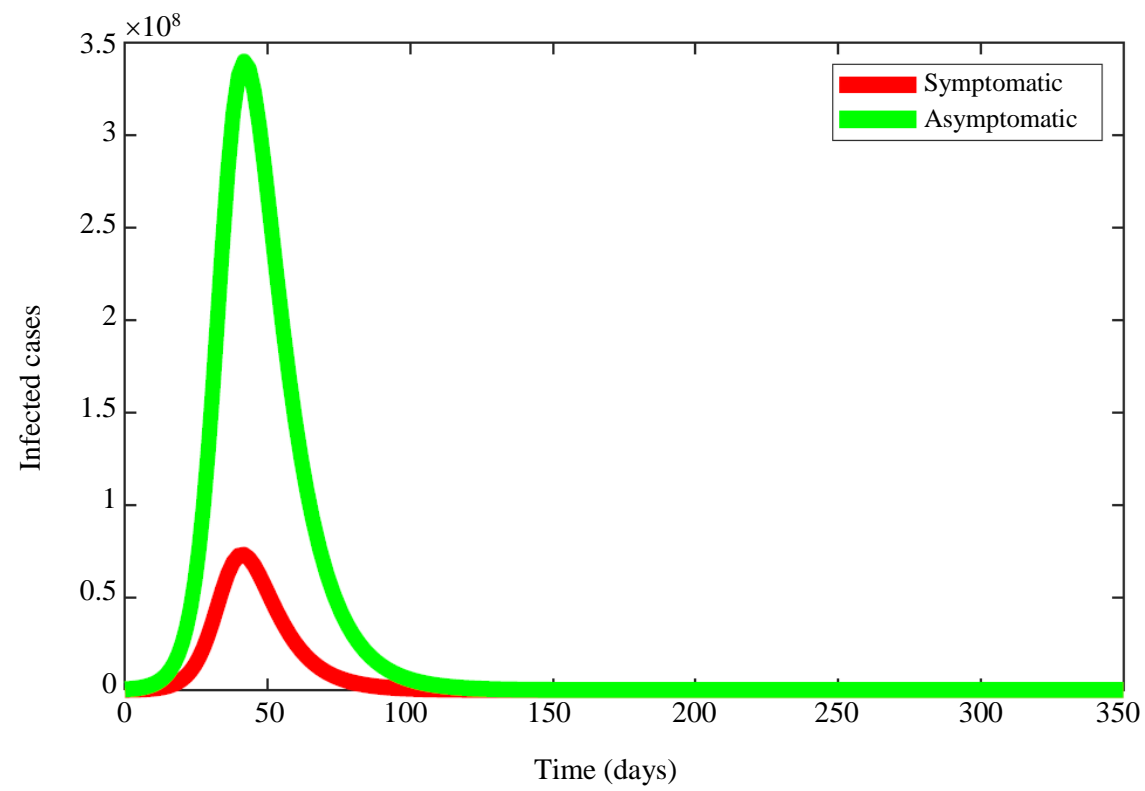

Fig. 14: Graphical simulation of the novel COVID-19 outbreak. Base model yields $R_{0}=2.5$ using $\beta=0.3125$ 


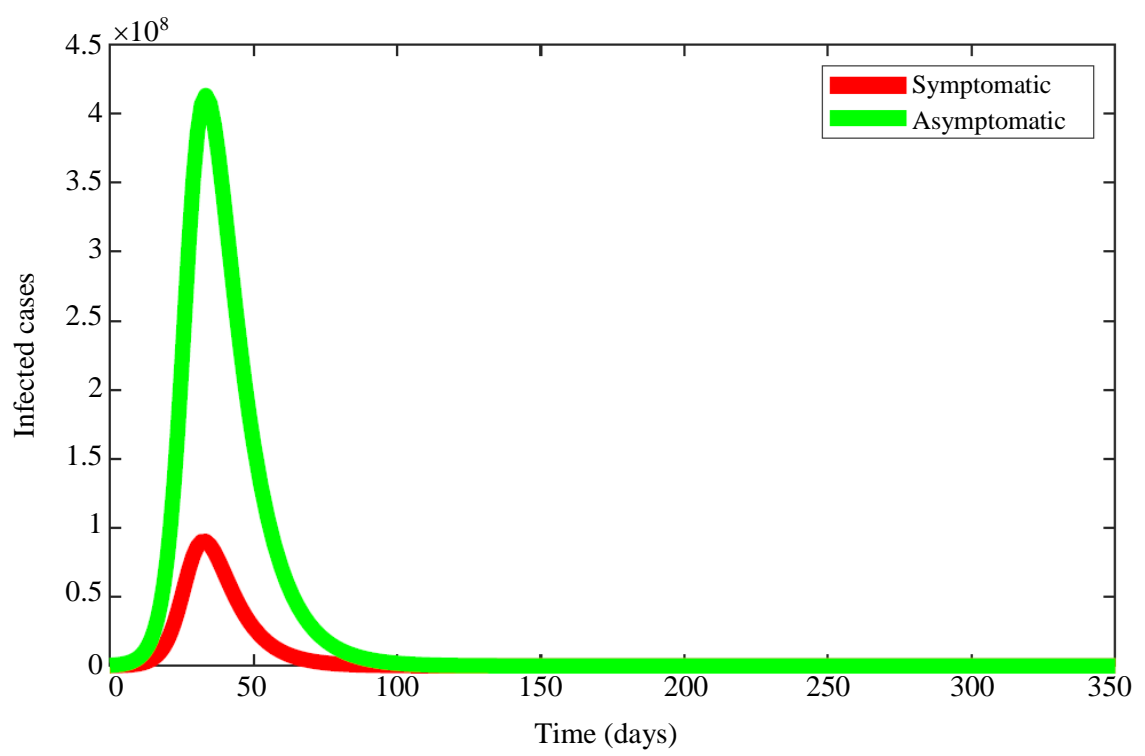

Fig. 15: Graphical simulation of the novel COVID-19 outbreak. Base model yields $R_{0}=3.0$ using $\beta=0.375$

A mean is a number that in some sense represents the central value of a set of numbers. Mathematical average of two or more numbers is called as mean. There are many ways to compute the mean of a series of numbers. We use the sum of the given numbers in the arithmetic mean method. Geometric mean is computed by the average of a set of products. The arithmetic mean which is also called as mean or average that was found by summing the numbers in the data and dividing that sum by the number of count. Generally, the data is the result from an observational study, experiment or a survey (Wikipedia, 2020b).

In the above graphs, the outbreak of the novel COVID-19 pandemic is plotted for different values of $R_{0}$ $=1.25,1.5,2.0,2.5$ and 3.0. The outbreak of the novel COVID-19 pandemic is also plotted for the mean value of the above ' $R_{0}$ '. In Fig. 16 , the mean ' $R_{0}$ ' was found to be 2.05 and the corresponding ' $\beta$ ' was found to be 0.25625 . Furthermore, ' $R_{0}$ ' does not remain constant for SARS-CoV-2 pandemic. Its value depends upon many factors such as environmental conditions; frequency of contact between the infected and susceptible individuals, measures taken to contain the spread, etc. The pandemic is predicted to end after 125 days from the present day, i.e., 28th July, 2020, using the current value of $\beta=0.25625$. The value of ' $R_{0}$ ' focuses light on the dynamics of the spread of disease. The mathematical models are used to determine the value of ' $R_{0}$ ' which also depends upon the other parameters used in mathematical modeling. Many popular presses have used ' $R_{0}$ ' which gave rise to misunderstandings related to its true meaning. Various mathematical models are used to calculate the value of ' $R_{0}$ '. Each of these models may give a different estimate the value of ' $R_{0}$ '. These values should be interpreted in the context of that model.

The value of ' $R_{0}$ ' can be used as a threshold, even if calculated with different methods. Whether the outbreak will die out or whether it will increase can be guessed using this number. If $R_{0}<1$, the outbreak will die out and if $R_{0}>1$, the outbreak will expand. In some cases, for some models, values of $R_{0}<1$ can still lead to selfperpetuating outbreaks. This is particularly problematic if there are intermediate vectors between hosts, such as malaria. Therefore, comparisons between values from the "Values of ' $R_{0}$ ' of well-known infectious diseases" table should be conducted with caution (Wikipedia, 2020b).

\section{Education Model}

A program including education would reduce the probability of transmitting an infection by half by increasing the awareness of people regarding maintaining social contact and improving personal hygiene.

We have not considered the vaccination but due to increased awareness between people, it would eventually give an effect of increased rate of vaccination to $10 \%$. Fear of the novel COVID-19 has the country under its grip. Many state governments have ordered the closure of schools and colleges, movie halls, gyms, malls, etc. Air traffic has fallen, which is indicative of reduced economic activity. Many such happenings and signs of destructive effects of the novel COVID-19 have a direct effect on the behaviour of every citizen. People have become more aware about this virus and its spread. People are finding and comprehending the ways which will protect themselves and their families from the impact of this virus 
(News18, 2020). We have plotted the graph from $15^{\text {th }}$ June, 2021 onwards considering the value of ' $\beta$ ' to be 0.128125 (Fig. 17). The pandemic is predicted to end after 700 days from the present day, i.e., 28th July, 2020.

The Government of India managed to quickly spread the awareness regarding this virus by using every possible mode of communication. Ringtones were applied to spread the awareness regarding this virus and how to contain its spread. Observing the impact of this virus in other countries, containment was the only option. Passengers coming from abroad were screened for infection. Combating other weapons which helped in fighting the spread of this virus are building infrastructure for the infected patients, increasing the testing facilities and screening people who possibly may have come in contact with the infected individual. To maintain the confidence of people in the system, it is necessary to ensure that the supply of masks, sanitizers and drugs is available to each one (Atkins, 2010; Rihan and Anwar, 2012; News18, 2020; BBC News, 2020b).

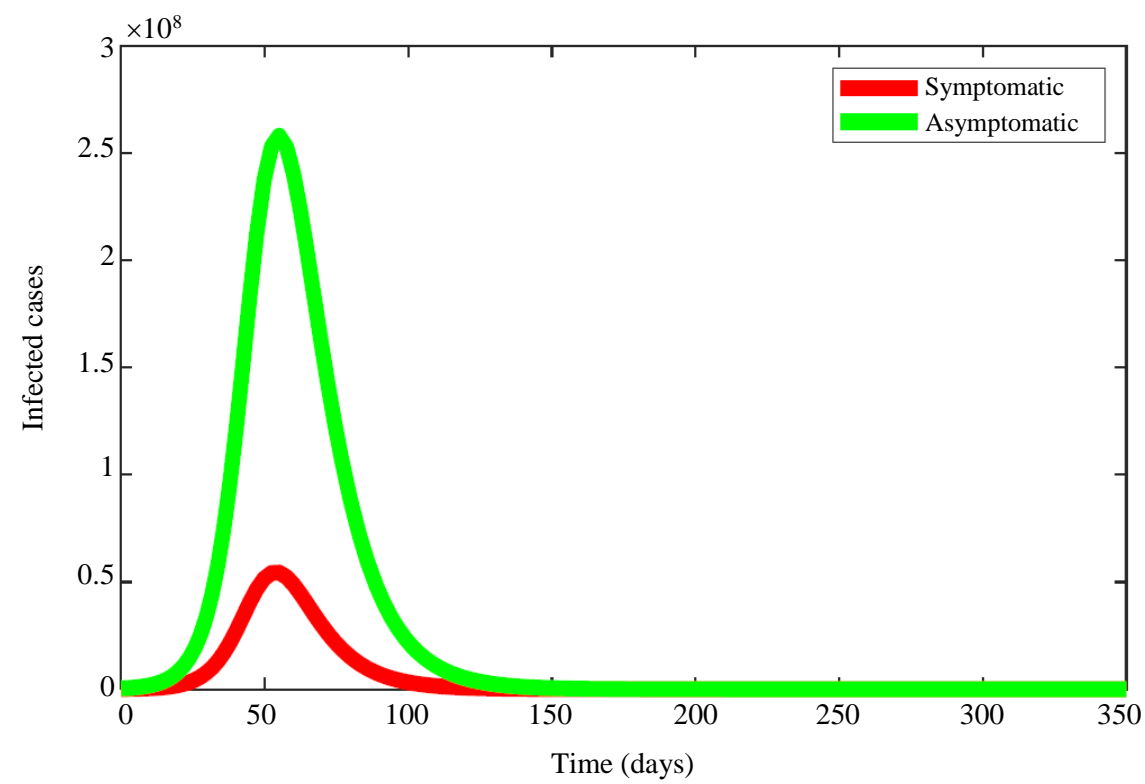

Fig. 16: Graphical simulation of the novel COVID-19 outbreak. Base model yields $R_{0}=2.05$ using $\beta=0.25625$

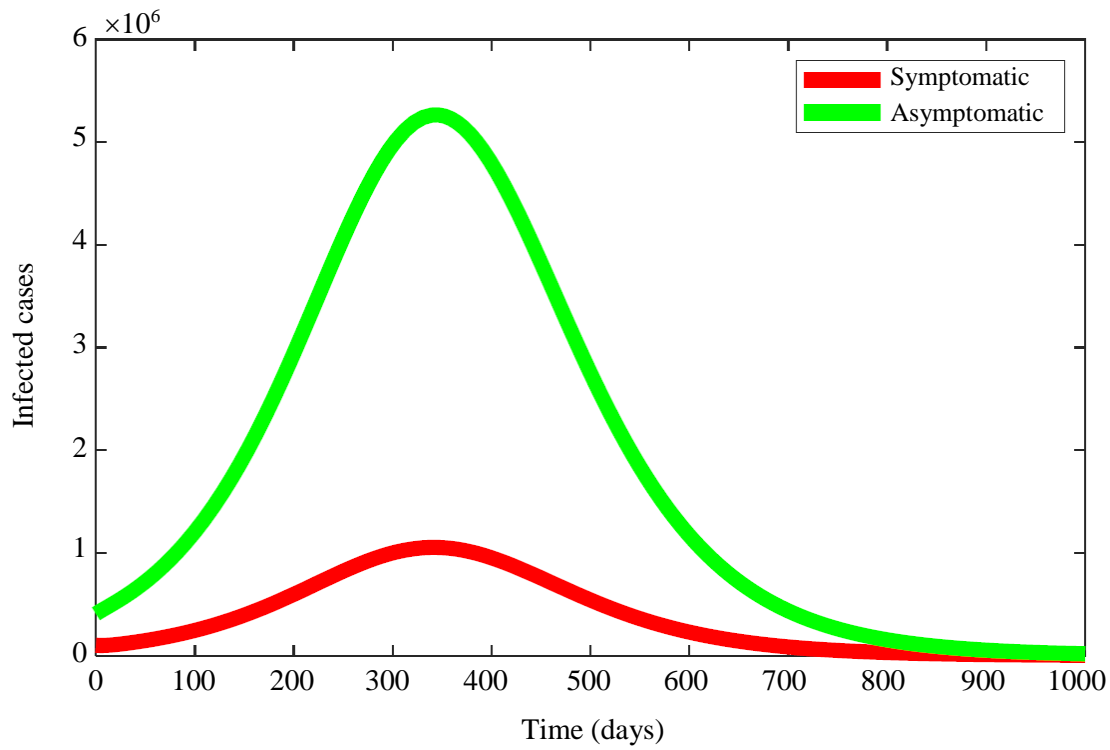

Fig. 17: Graphical simulation of the novel COVID-19 outbreak. Education model yields $R_{0}=1.025$ using $\beta=0.128125$ 


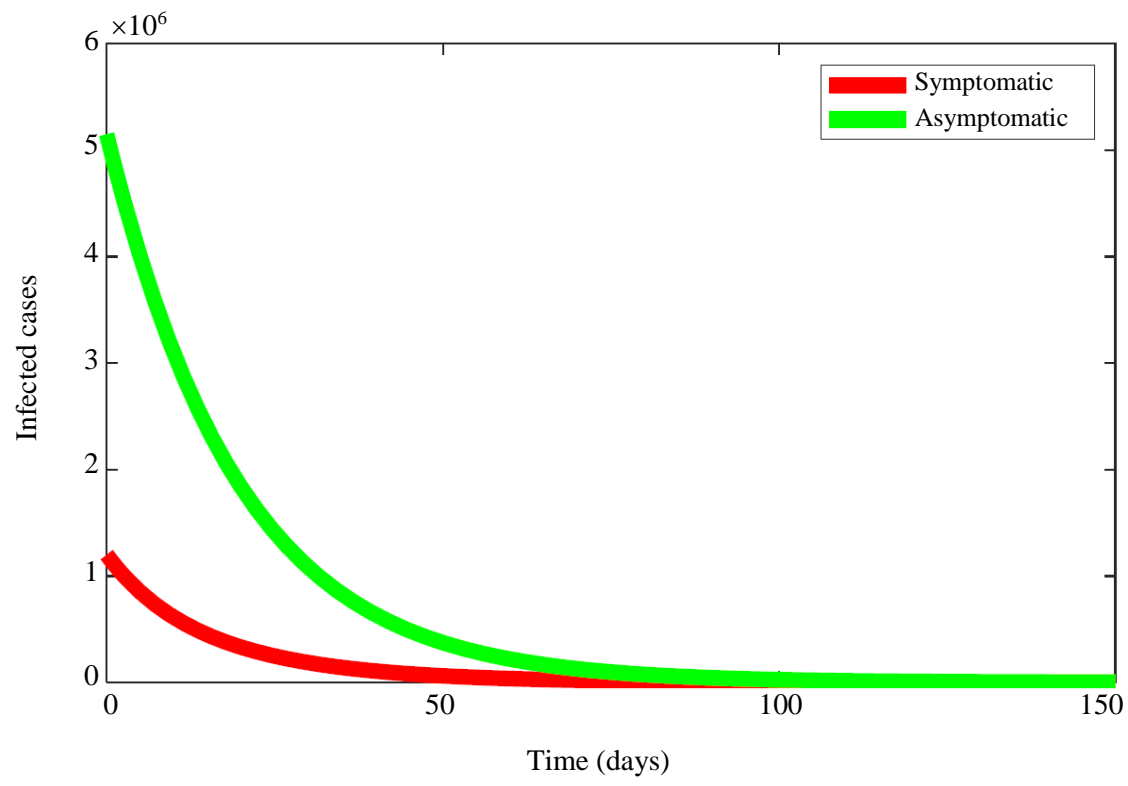

Fig. 18: Graphical simulation of the novel COVID-19 outbreak. Education and vaccination model yields $R_{0}=1.025$ Assuming $61 \%$ of the population is vaccinated

\section{Education and Vaccination Model}

Generally, a vaccine may take years to develop. According to the experts, we can expect the vaccine to be available by the end of February, 2021. We have assumed that $61 \%$ of the population of India would be vaccinated up to 15 th June, 2021. Therefore, we have plotted the graph from 15th June, 2021and onwards considering the value of ' $\beta$ ' to be 0.128125 (Fig. 18). The pandemic is predicted to end after 100 days from 15th June, 2021. The parameter, ' $V_{0}$ ' was considered $8,28,584,757$. Research on the vaccine for the novel COVID-19 is happening at breakneck speed. At present, around 140 vaccines are in their early stage of development and about 24 numbers of vaccines are in their clinical trials on people. The vaccine developed by Oxford University is reported to trigger the immunity system to respond against the virus. According to the deal signed with AstraZeneca, 100 million doses will be supplied in the UK alone. A study performed in May in the United States of America resulted in the production of antibodies that could neutralize the novel COVID-19 in every eight patients taking part in the study. Other completely new approaches to vaccine development are in human trials (Ranjan, 2020).

Many research groups have designed the vaccine that has the potential to fight the novel COVID-19, but still a lot of work is left to do. These researchers must show that their vaccine is safe to be injected in the body. The vaccine should cause an immune response, which will work as a shield to the people and prevent them from getting sick. Furthermore, the vaccine should be able to be produced on a large scale so that billions of doses can be developed. The medicine regulatory frame must approve it before it is actually provided to the population. At last, the most difficult challenge is to immunize the world's population at a substantial scale (Atkins, 2010; The Times of India, 2020a; Deb and Majumdar, 2020).

City Wise Discussion on Transmission of the Novel Coronavirus Disease (COVID-19) Pandemic in India using Stochastic Susceptible (S)-Infected (Symptomatic) (I $I_{S}$ ) Infected (Asymptomatic) $\left(I_{N}\right)$ Recovered (R) $\left(S I_{S} I_{N} R\right)$ Model

\section{Stochastic Model Forecasting the Outbreak of the Novel COVID-19 in Delhi}

The first case of the novel COVID-19 pandemic in the Indian capital, Delhi, was reported on 02nd March, 2020. Delhi has the third highest number of confirmed cases of the novel COVID-19 pandemic in India after Maharashtra and Tamil Nadu. The total 1,34,403 infected people were reported on 31st July, 2020, including 3,936 deaths and 1,19,724 recovered patients. On 22nd March, 2020, Delhi observed a 14 h voluntary public curfew named Janata curfew along with 75 districts in India at the directive from Honourable Prime Minister of India, prior to his order of nationwide lockdown from 24th March, 2020. Thousands of migrants from Uttar Pradesh and Bihar gathered at the bus stand and railway station to head back to their native places. More than 3,000 people from a social gathering were quarantined after 
suspected contact with infected people, including foreigners. The Delhi government announced an operation known as SHIELD, i.e., S-sealing the area immediately, $\mathbf{H}$-home quarantine to the people living in the area, I refers to isolation and contact tracing of people, $\mathbf{E}$ refers to essential supply of commodities, $\mathbf{L}$ refers to local sanitization and D-door to door health check upon order to restrict the spread of the novel COVID-19 pandemic in the containment zones or the hotspots around the state (Business Today, 2020).

The novel COVID-19 pandemic was widely spread in Dilshad Garden, from where the first success came. On 10th April, the government announced that Operation SHIELD was working quite successfully. This area became the novel COVID-19 free after implementation of the SHIELD operation. This operation also emerged successful in Vasundhara Enclave and Khichripur (The Times of India, 2020b; Wikipedia, 2020c).

An order was issued making 7 days home quarantine compulsory for all asymptomatic individuals who travelled into the city through domestic transport. Individuals who showed symptoms were immediately tested for the novel COVID-19 and were quarantined at the institutional level till the results were declared. If persons were found positive, they were moved to a hospital or any novel COVID-19 Care Centre. If they were found negative, they were instructed to follow the 14 days home quarantine. The details on containment zones and hotspots, testing facilities, key locations, epass procurement and relevant frequently asked questions were posted on this website. The information also included the locations of grocery shops, temporary relief centres and hunger relief centres in Delhi. On 24th June, 2020, the Delhi government set up the world's largest temporary novel COVID-19 care hospital of
10,000 beds, as the country grapples with rapidly rising cases (Wikipedia, 2020c).

The value of ' $R_{0}$ ' focuses light on the dynamics of the spread of disease and thus suitable graphs have been plotted using the above inputs to discuss the spread of the novel COVID-19 pandemic in the National Capital Region of Delhi (Fig. 19) using stochastic mathematical models based on the modified $S I_{S} I_{N} R$ transmission network model. With an increase in the values of ' $\beta$ ' and thus ' $R_{0}$ ', the peaks of infected cases (both symptomatic and asymptomatic cases) as well as the end of SARSCoV-2 pandemic are attended soon after $31^{\text {st }}$ July, 2020 as discussed in Fig. 19 . The first case $\left(\beta=0.15625 ; R_{0}=\right.$ $1.25)$ is more appropriate as it shows the peak of the novel COVID-19 pandemic in December, 2020 (Fig. 19a). The curve shows the zero case of pandemic by the end of March, 2021.

\section{Stochastic Model Forecasting the Outbreak of the Novel COVID-19 in Pune}

On 09th March, 2020, a couple who arrived in India from Dubai were tested positive for the novel COVID-19 and were the first confirmed case from Maharashtra. Three more people who were in contact with this couple were also tested positive on the next day. All five of them were taking treatment in Naidu Hospital. After 9 days on 18th March, 2020, the woman was tested positive in Pune. This woman had earlier travelled to France and Netherlands. An old woman who was in her late 60's was linked with a confirmed case also tested positive. The number of positive cases rose to 45 after cases were found in Pimpri-Chinchawad and Ratnagiri. The case found in Pimpri-Chinchwad had travelled to Singapore, Philippines and Sri Lanka. The latter case from Ratnagiri earlier travelled to Dubai.
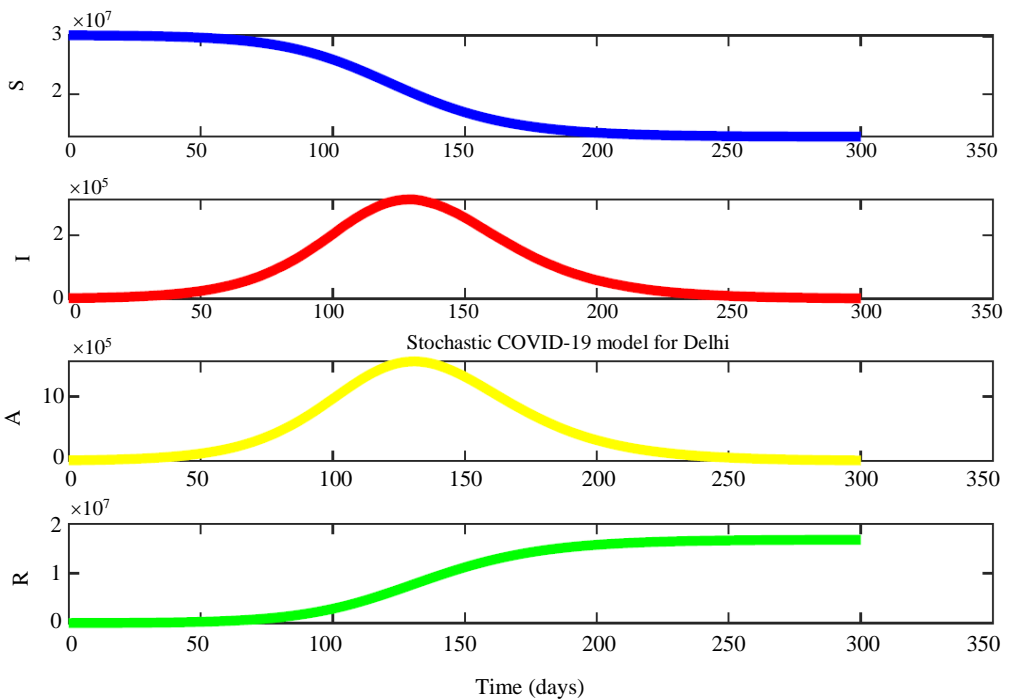

(a) 
Eva Gupta et al. / American Journal of Infectious Diseases 2020, 16 (4): 135.170 DOI: 10.3844/ajidsp.2020.135.170
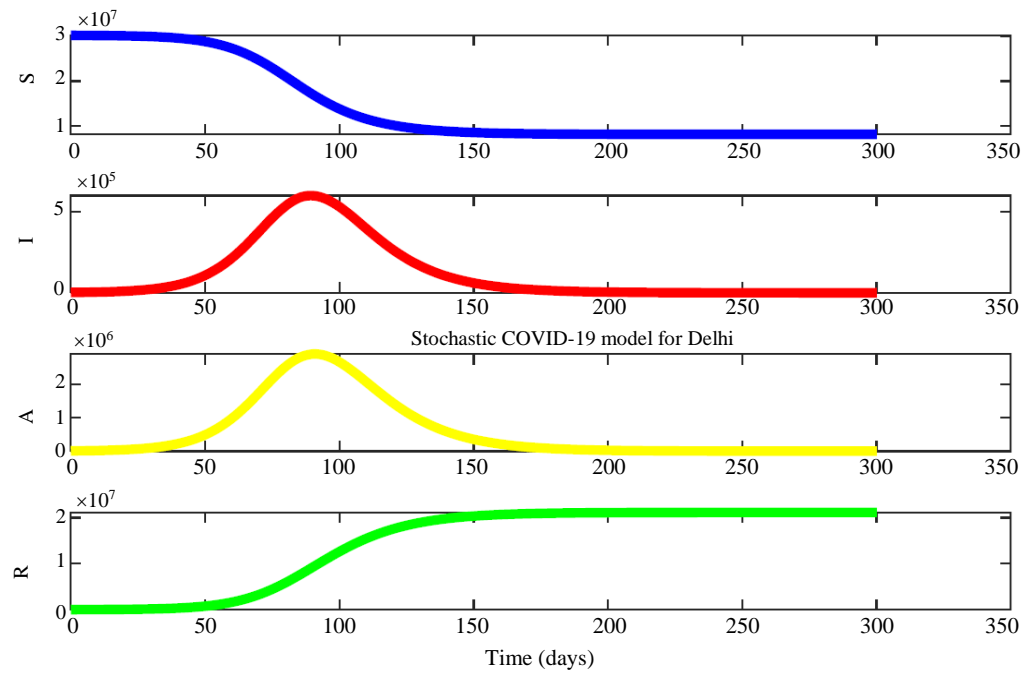

(b)
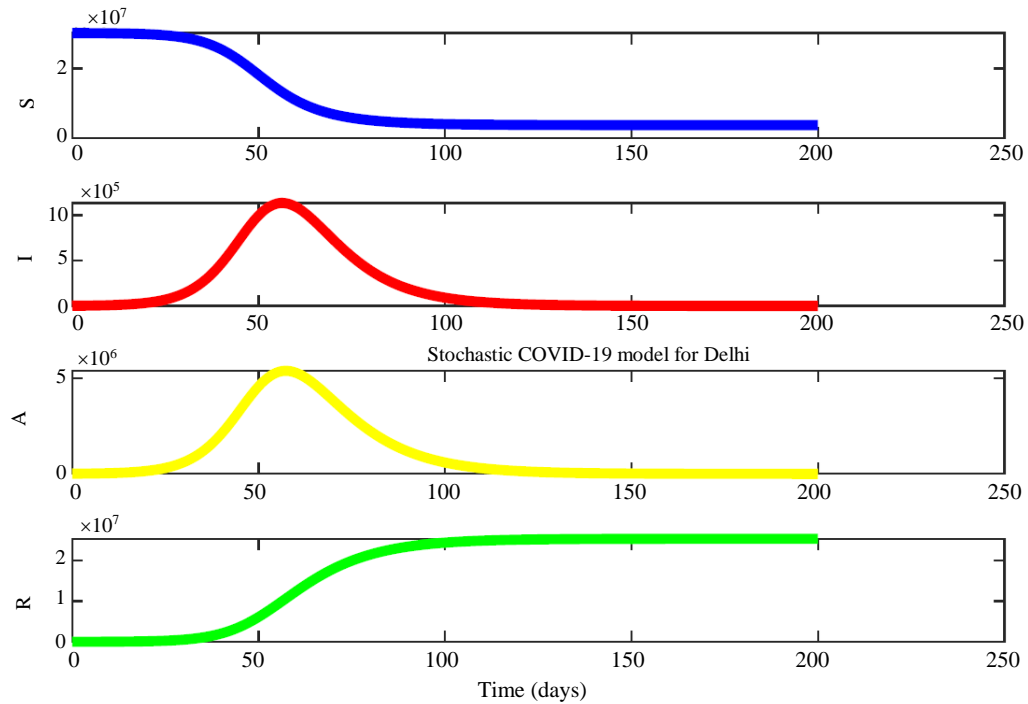

(c)
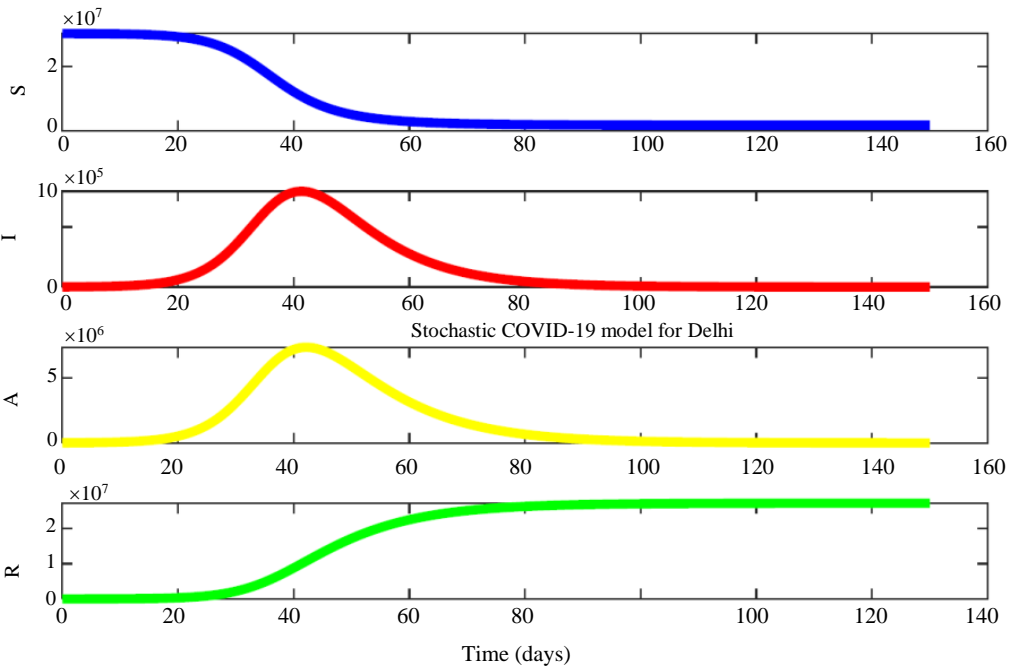

(d) 

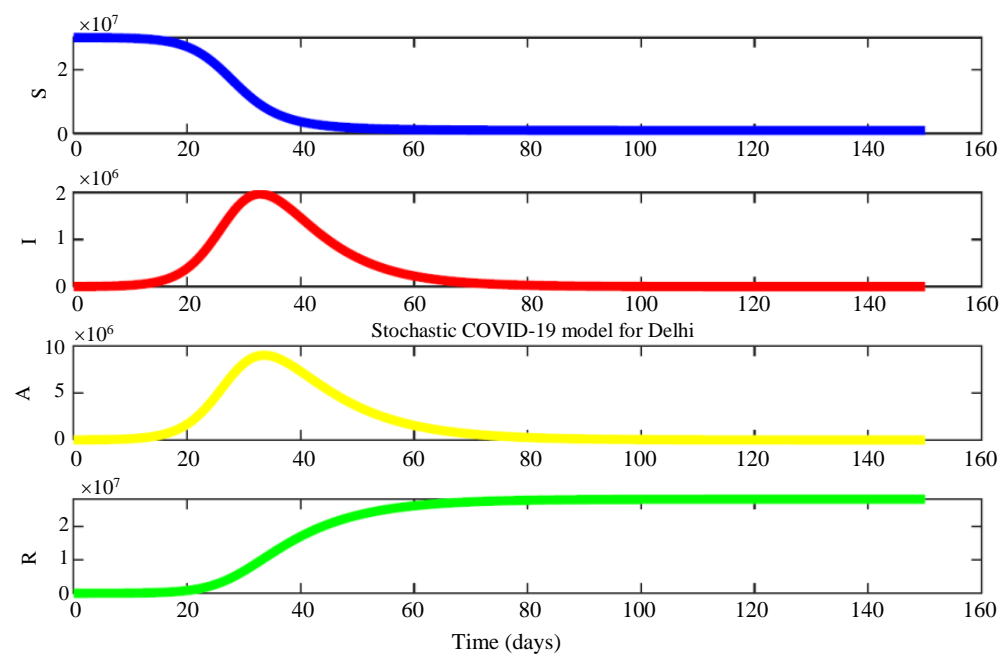

(e)

Fig. 19: The stochastic $S_{S} I_{N} R$ model of the novel COVID-19 for National Capital Region of Delhi considering sustained lockdown of 61 days, i.e., 25th March, 2020-31st May, 2020, unlock 0.1 and 0.2, as well as weekly lockdown in July, 2020 including night curfew and implementation of effective quarantine periods for travelers. (a) $S I_{S} I_{N} R$ model based on the novel stochastic approach $\left(\beta=0.15625 ; R_{0}=1.25\right)$. (b) $S I_{S} I_{N} R$ model based on the novel stochastic approach $\left(\beta=0.1875 ; R_{0}=1.5\right.$. (c) $S I_{S} I_{N} R$ model based on the novel stochastic approach $\left(\beta=0.25 ; R_{0} 2.0\right)$. (d) $S I_{S} I_{N} R$ model based on the novel stochastic approach $(\beta$ $\left.=0.3125 ; R_{0}=2.5\right)$. (e) $\operatorname{SI}_{S} I_{N} R$ model based on the novel stochastic approach $\left(\beta=0.375 ; R_{0}=3.0\right)$. Note: The parameters such as $S, I, A$ and $R$ are used to express susceptible, infected (symptomatic), infected (asymptomatic) and recovered patients, respectively

The population of Pune is estimated to be 6.8 million while Mumbai's population is much greater. Mumbai had the largest number of positive cases in Maharashtra as well as in India. The total positive cases in Mumbai were 84,524. On 03rd July, 2020, Pune city had $25 \%$ cases against Mumbai and a fatality rate of 3.32. Mumbai was reported to have a fatality rate $5.85 \%$ (India Today, 2020b).

Although Pune has better numbers as compared to Mumbai, it is on the same path as that of Mumbai. The total of infected people reported on 31st July, 2020 is 28,142 consisting of 872 deaths and 13,406 recoveries (The Hindustan Times, 2020a; NDTV, 2020).

As the value of ' $R_{0}$ ' focuses light on the dynamics of the spread of disease and thus suitable graphs have been plotted using the above inputs to discuss the spread of the novel COVID-19 pandemic in the Pune region of the Maharashtra state (Fig. 20) using stochastic mathematical models based on the modified $S I_{S} I_{N} R$ transmission network model. Again, with an increase in the values of ' $\beta$ ' and thus ' $R_{0}$ ', the peaks of infected cases (both symptomatic and asymptomatic cases) as well as the end of SARS-CoV-2 pandemic are attended soon after 31st July, 2020 as discussed in Fig. 20. For Pune city, the first case $\left(\beta=0.15625 ; R_{0}=1.25\right)$ is more appropriate as it shows the peak of the novel COVID-19 pandemic in December, 2020 (Fig. 20a). The curve shows the zero case of pandemic by the end of March, 2021.

\section{Stochastic Model Forecasting the Outbreak of the Novel COVID-19 in Mumbai}

Mumbai was the worst affected city in India, with about 100,000 cases. About half of the cases in the state emerged from the Mumbai Metropolitan Region (MMR). On 11th March, 2020, two people in Mumbai who were linked to the Pune couple tested positive. To prohibit the spread of coronavirus, various measures were taken by the Navi Mumbai civic body. According to the orders, roadside stores and commercial shops, outlets near public places and crowded places such as railway stations were closed on alternate days till the end of the March. Many measures to control the crowd and prohibit the spread of the coronavirus were taken by the administrative body. One of the measures to control the crowd and contain the spread of coronavirus was the order issued by the municipal commissioner to close shops, stores and roadside outlets, near footpaths and near crowded stations such as CBD Belapur, Nerul, Vashi, Sanpada, Kopar Khairane, Ghansoli and Airoli on alternate days (The Hindu, 2020a; The Hindustan Times, 2020b; Kumar, 2020).

Mumbai saw a delay in the diagnosis of the patients because the testing rate in Mumbai was low initially. Citizens wishing to be tested for the novel COVID-19 were sent back by municipal doctors. The same case was seen in Pune too. Due to the delay, the patient's condition was critical. This increased the number of serious patients and deaths. 
Eva Gupta et al. / American Journal of Infectious Diseases 2020, 16 (4): 135.170 DOI: $10.3844 /$ ajidsp.2020.135.170
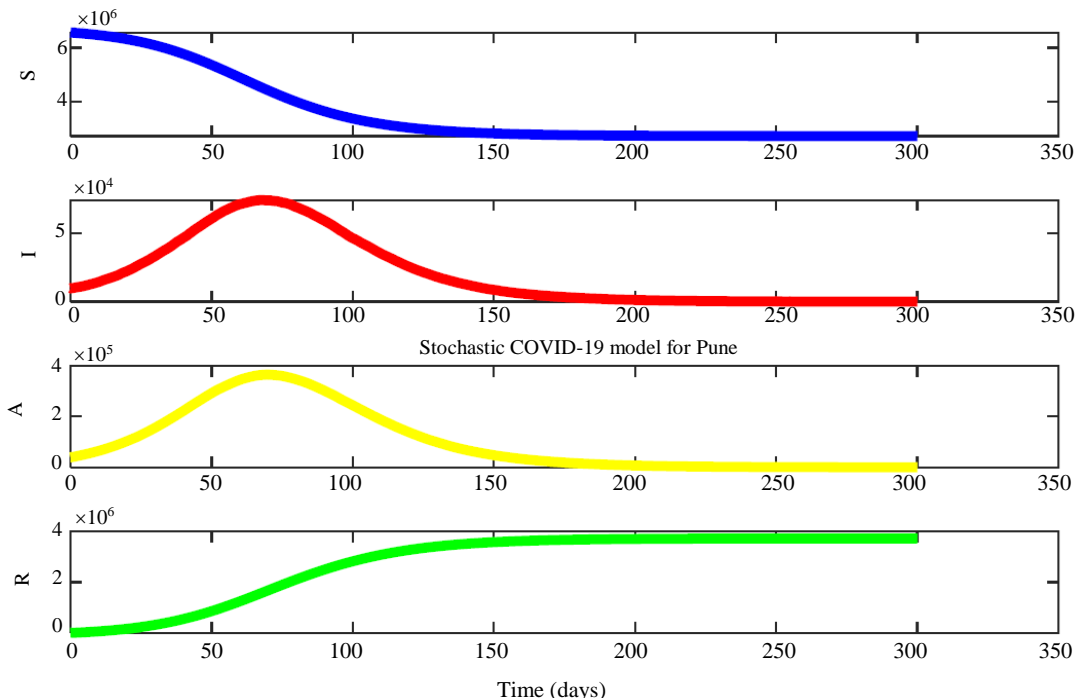

(a)
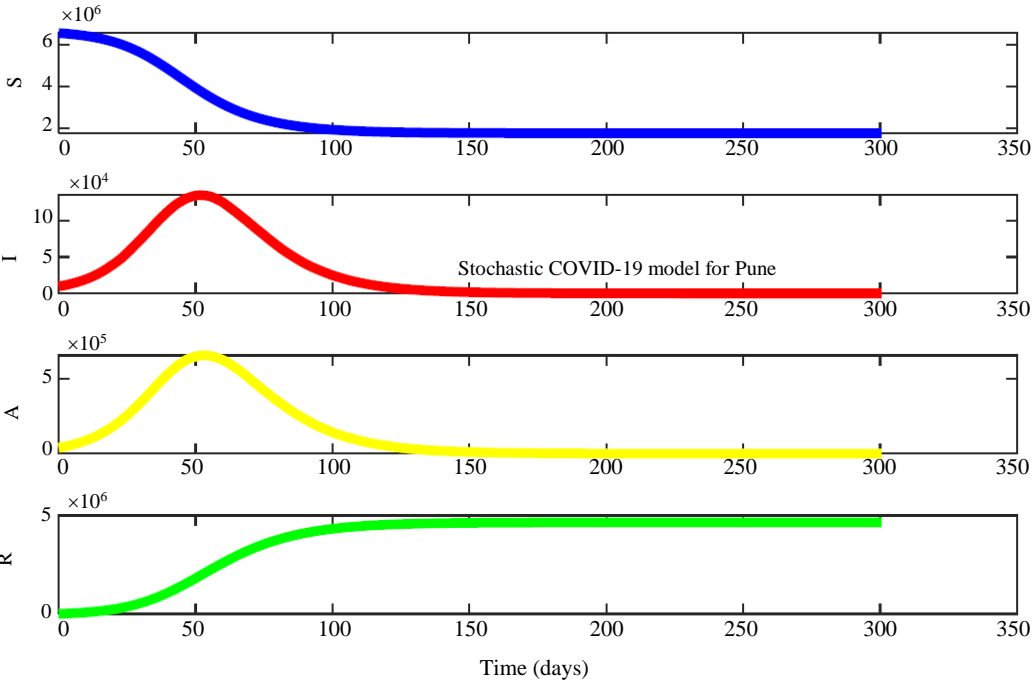

(b)
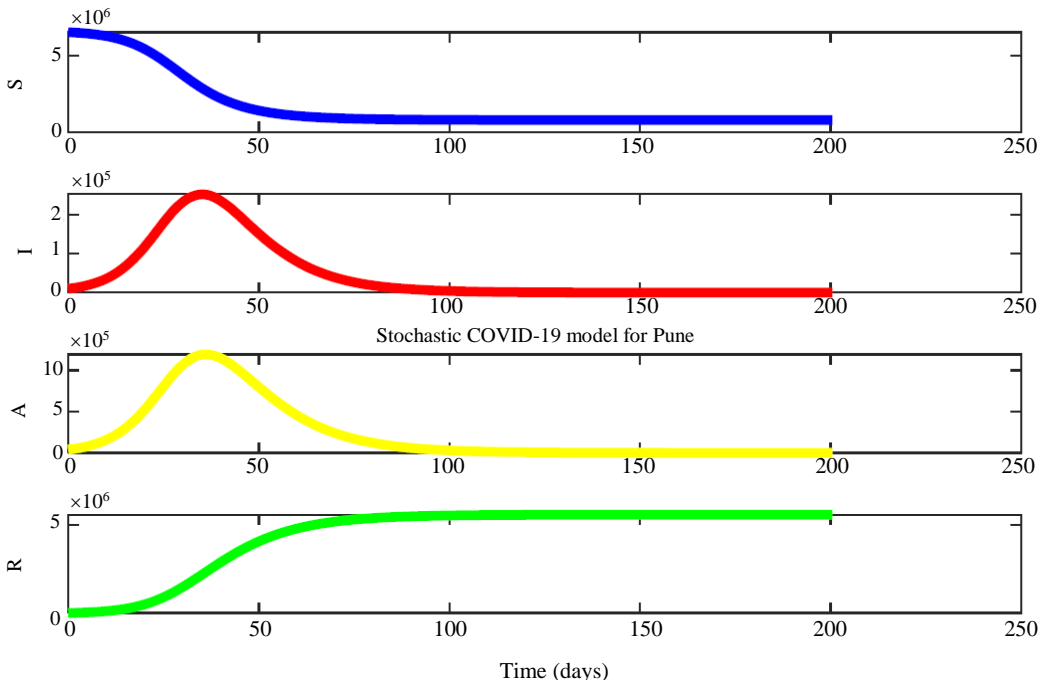

(c) 

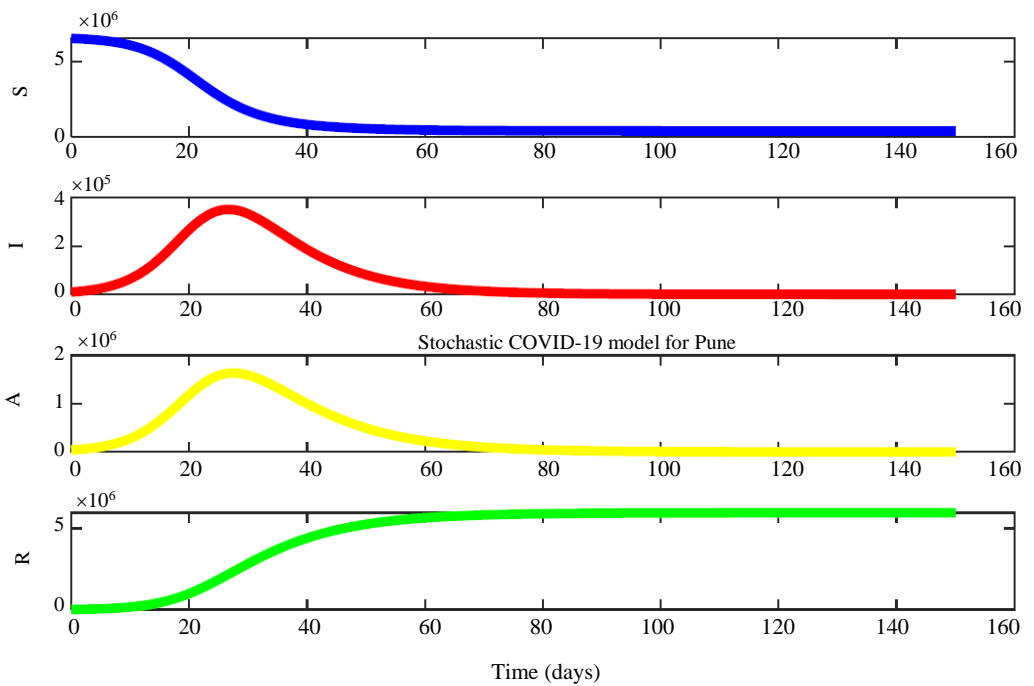

(d)
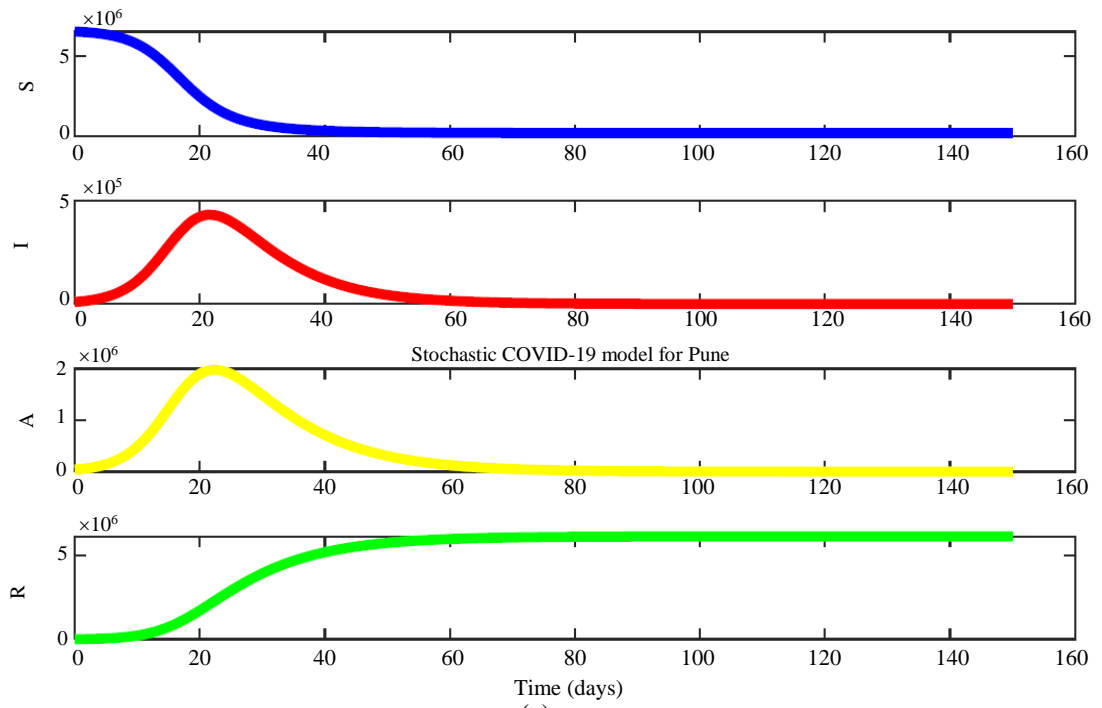

(e)

Fig. 20: The stochastic $S I_{S} I_{N} R$ model of the novel COVID-19 for Pune considers a sustained lockdown of 61 days, i.e., 25th March, 2020-31st May, 2020, unlock 0.1 and 0.2, as well as weekly lockdown in July, 2020 including night curfew and implementation of effective quarantine periods for travelers. (a) $S I_{S} I_{N} R$ model based on the novel stochastic approach $(\beta=$ $\left.0.15625 ; R_{0}=1.25\right)$. (b) $S_{S} I_{N} R$ model based on the novel stochastic approach $(\beta=0.1875 ; R 0=1.5)$. (c) $\operatorname{SI}_{S} I_{N} R$ model based on the novel stochastic approach $\left(\beta=0.25 ; R_{0}=2.0\right)$. (d) $S I_{S} I_{N} R$ model based on the novel stochastic approach $(\beta=0.3125$; $\left.R_{0}=2.5\right)$. (e) $S I_{S} I_{N} R$ model based on the novel stochastic approach $\left(\beta=0.375 ; R_{0}=3.0\right)$. Note: The parameters such as $S, I$, $A$ and $R$ are used to express susceptible, infected (symptomatic), infected (asymptomatic) and recovered patients, respectively

The total of infected people reported on 31st July, 2020 is $1,14,284$, consisting of 6,353 deaths. The discussion is made on the basis of the value of ' $R_{0}$ ' as it helps to understand the dynamics of the spread of disease and thus suitable graphs have been plotted using the above inputs to discuss the spread of the novel COVID-19 pandemic in the Mumbai region of the Maharashtra state (Fig. 21) using stochastic mathematical models based on the modified $S I_{S} I_{N} R$ transmission network model. As discussed, with the increase in the values of ' $\beta$ ' and thus ' $R_{0}$ ', the peaks of infected cases (both symptomatic and asymptomatic cases) as well as the end of SARS-CoV-2 pandemic are attended soon after 31st July, 2020. For Mumbai city, the first case $\left(\beta=0.15625 ; R_{0}=1.25\right)$ is more appropriate in the current scenario as it shows the peak of the novel COVID-19 pandemic in December, 2020 (Fig. 21a). The curve shows the zero case of pandemic by the end of March, 2021. 
Eva Gupta et al. / American Journal of Infectious Diseases 2020, 16 (4): 135.170 DOI: 10.3844/ajidsp.2020.135.170
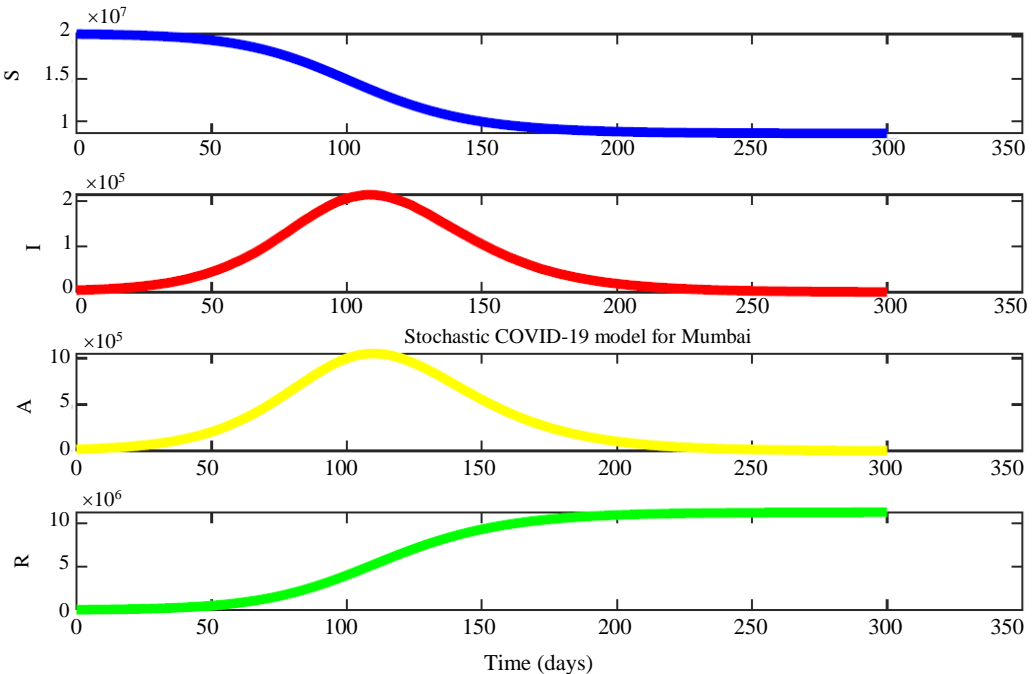

(a)
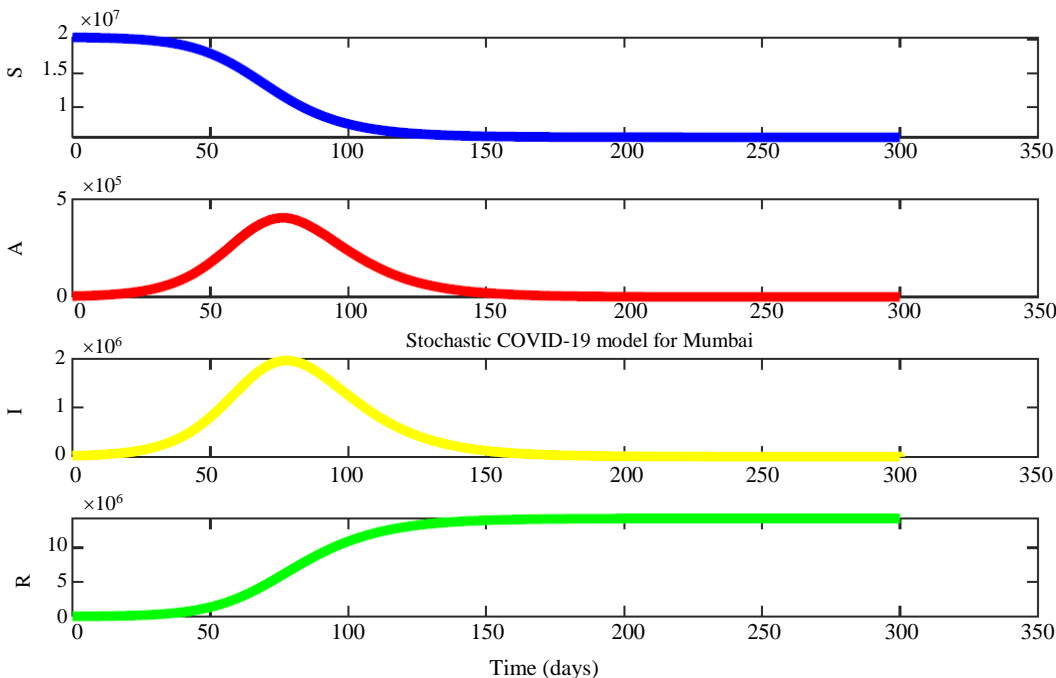

(b)
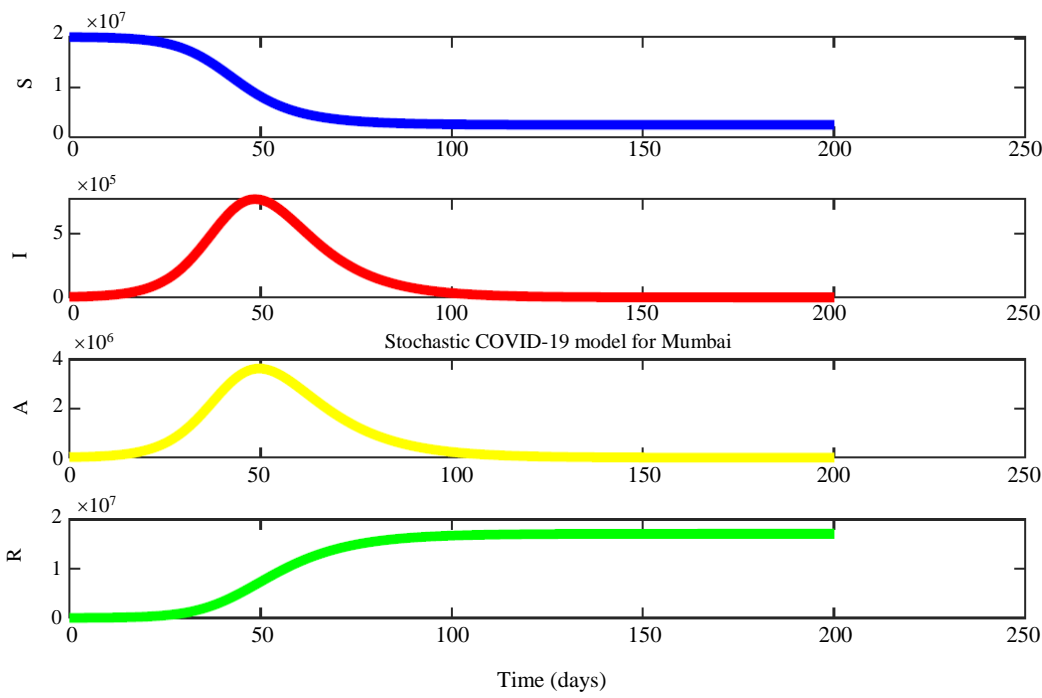

(c) 

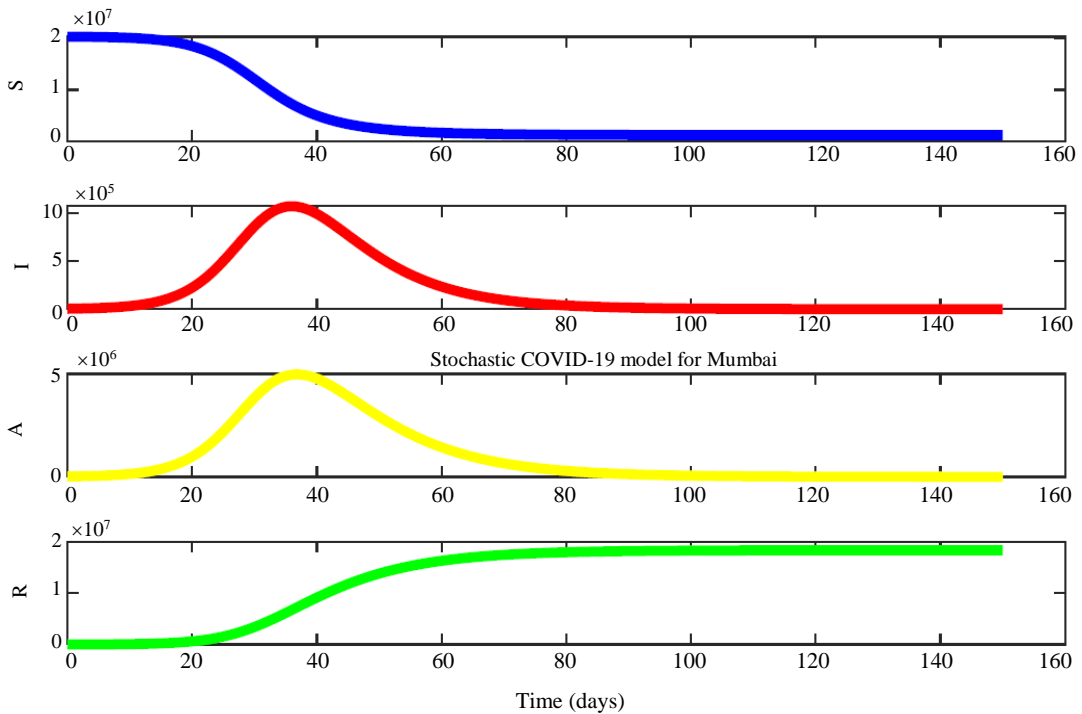

(d)
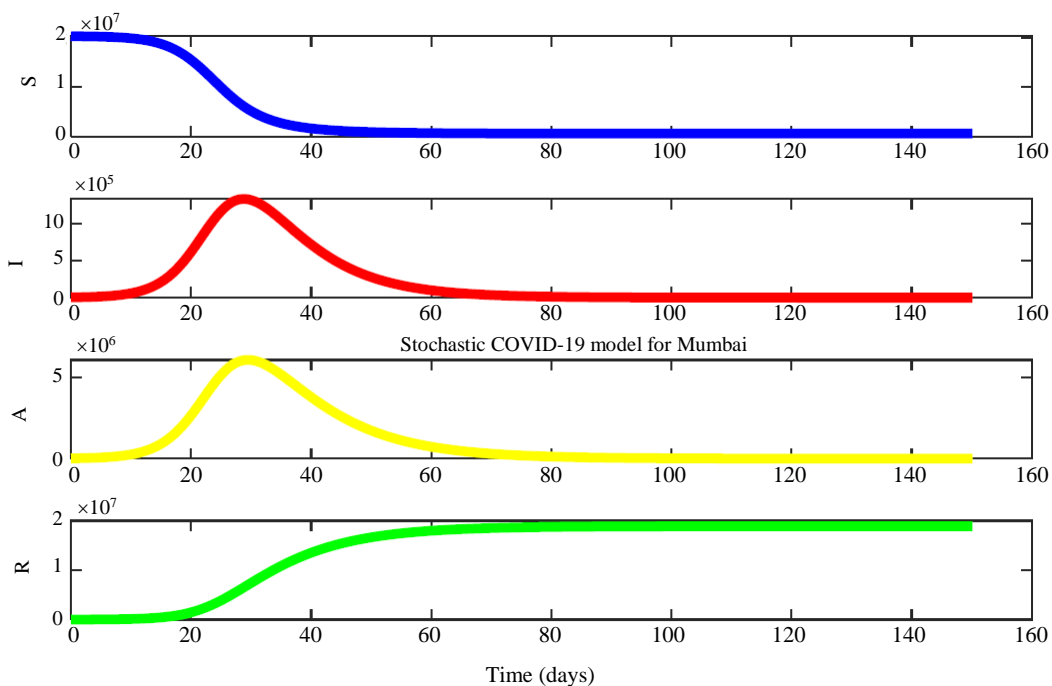

(e)

Fig. 21: The stochastic $S I_{S} I_{N} R$ model of the novel COVID-19 for Mumbai considers a sustained lockdown of 61 days, i.e., 25th March, 2020-31st May, 2020, unlock 0.1 and 0.2, as well as weekly lockdown in July, 2020 including night curfew and implementation of effective quarantine periods for travelers. (a) $S I_{S} I_{N} R$ model based on the novel stochastic approach $(\beta=$ $\left.0.15625 ; R_{0}=1.25\right)$. (b) $S I_{S} I_{N} R$ model based on the novel stochastic approach $\left(\beta=0.1875 ; R_{0}=1.5\right)$. (c) $S I_{S} I_{N} R$ model based on the novel stochastic approach $\left(\beta=0.25 ; R_{0}=2.0\right)$. (d) $S I_{S} I_{N} R$ model based on the novel stochastic approach $(\beta=0.3125$; $\left.R_{0} 2.5\right)$. (e) $S I_{S} I_{N} R$ model based on the novel stochastic approach $\left(\beta=0.375 ; R_{0}=3.0\right)$. Note: The parameters such as $S, I, A$ and $R$ are used to express susceptible, infected (symptomatic), infected (asymptomatic) and recovered patients, respectively

\section{Stochastic Model Forecasting the Outbreak of the Novel COVID-19 in Chennai}

Tamil Nadu has the second highest number of confirmed cases in India after Maharashtra. Three cases were initially reported in Chennai. The first case was of an old man who was in his mid 60's. He travelled to New Zealand and came back in India. He was treated at a private hospital. Second case of an old lady was found in her mid 50's. She was admitted to Kilpauk Medical College Hospital. The third case was a 25-year-old man who had travelled to London and came back to India. He was been treated at Rajiv Gandhi Government Hospital. Furthermore, 17 cases in Tamil Nadu were reported positive, which also included two foreign nationals. All districts of the state were suffering from the novel COVID-19 pandemic. Chennai was the worst affected. More than fifty percent of the total confirmed cases in Tamil Nadu were from Chennai. 
Eva Gupta et al. / American Journal of Infectious Diseases 2020, 16 (4): 135.170 DOI: 10.3844/ajidsp.2020.135.170
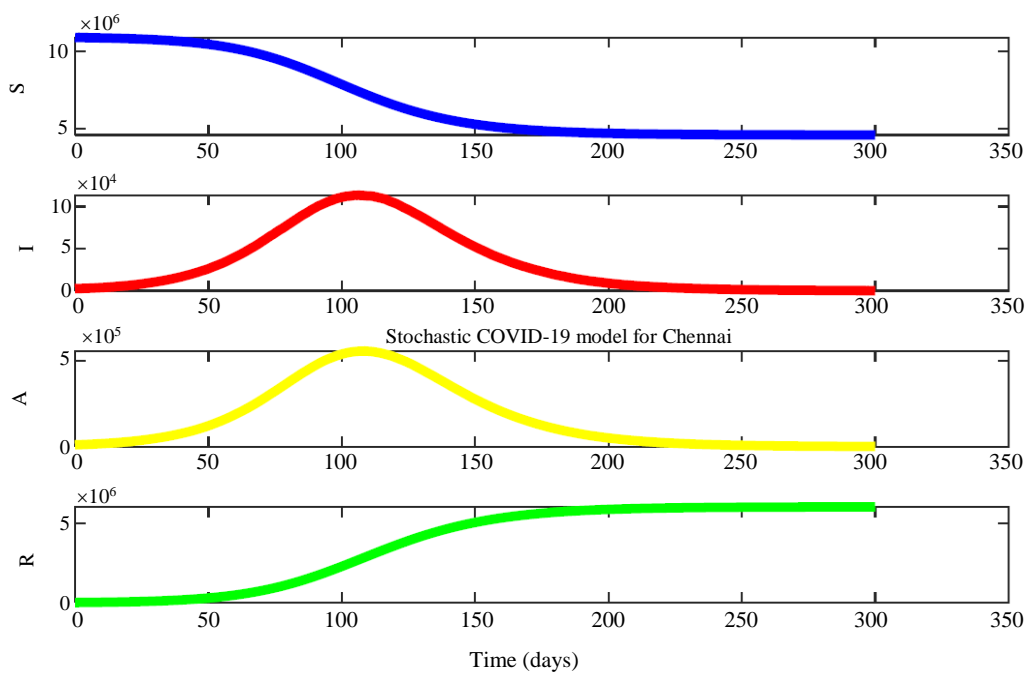

(a)
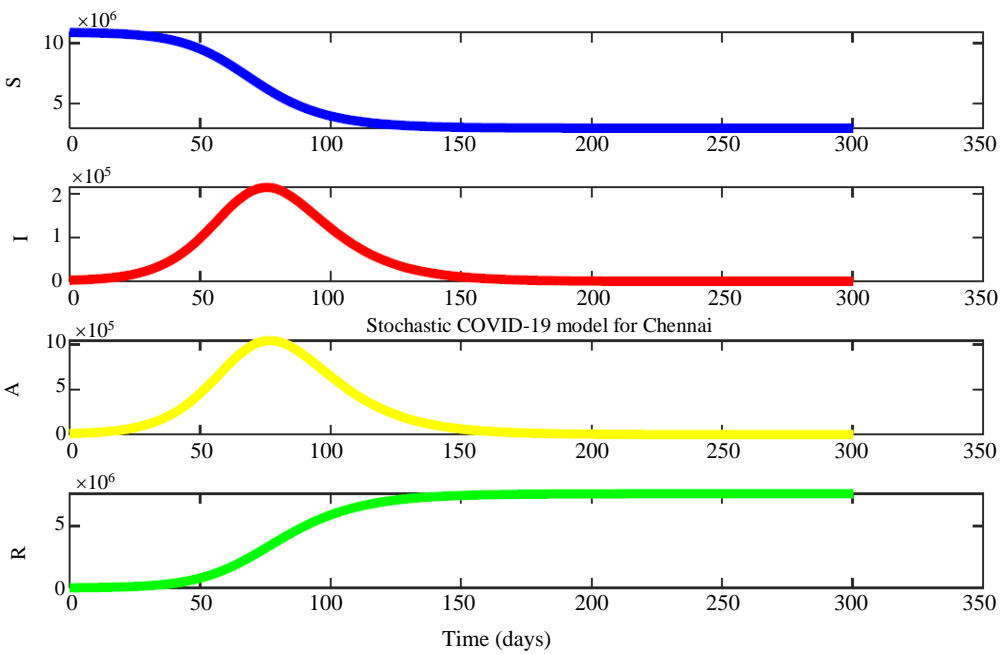

(b)
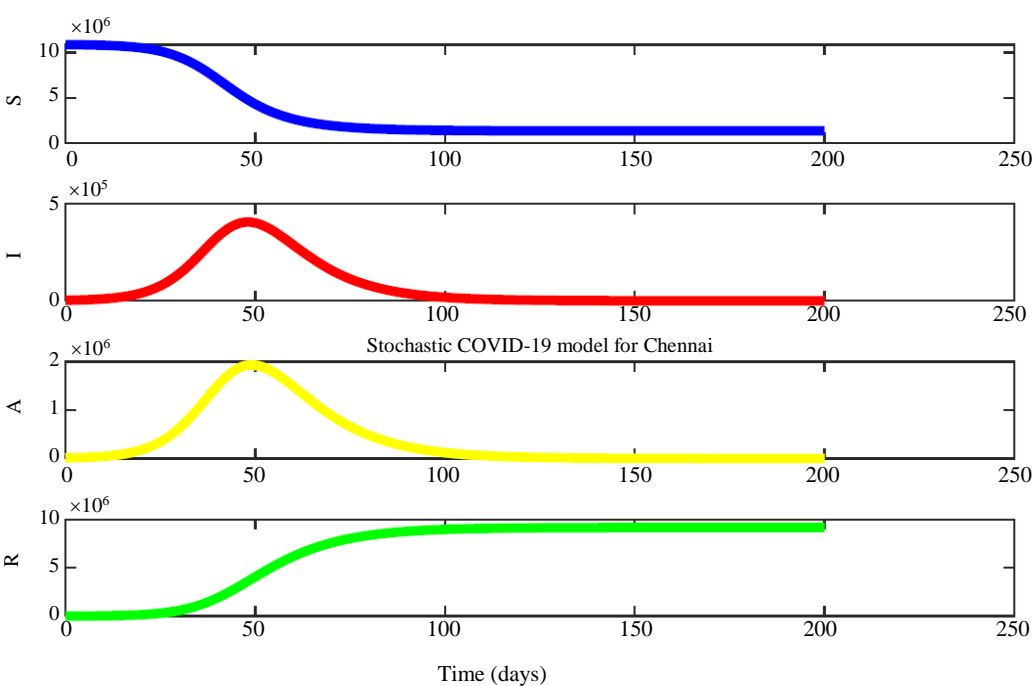

(c) 

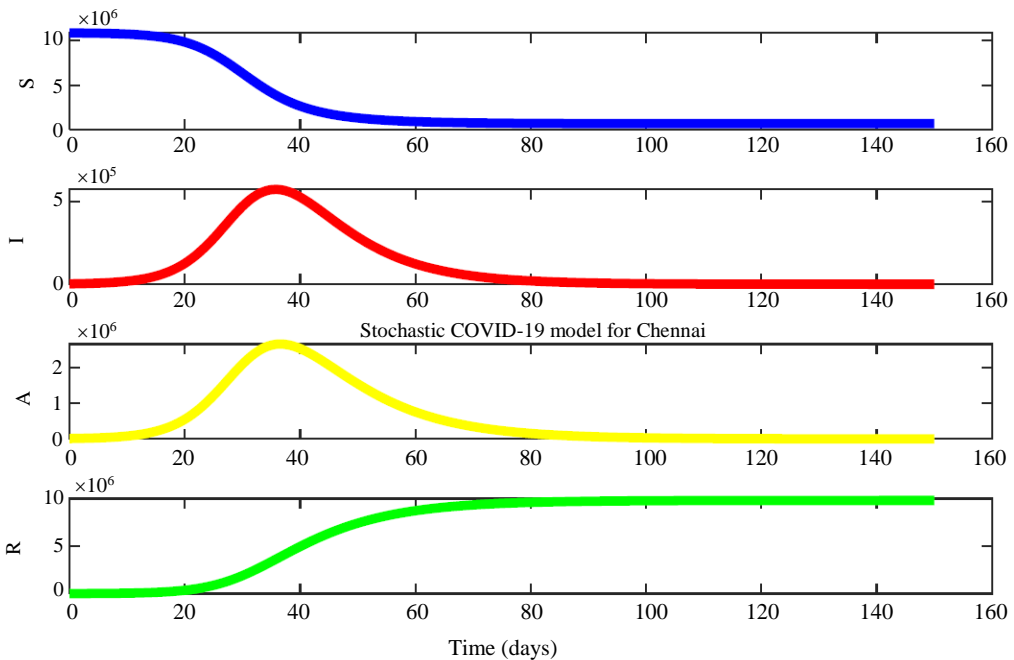

(d)
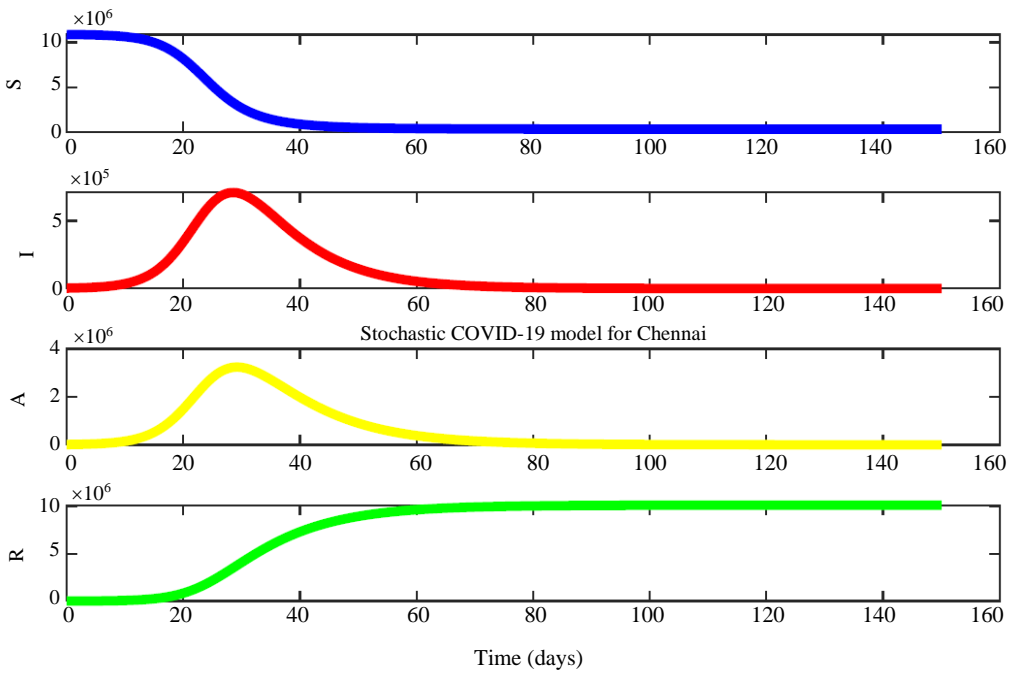

(e)

Fig. 22: The stochastic $S I_{S} I_{N} R$ model of the novel COVID-19 for Chennai considers a sustained lockdown of 61 days, i.e., 25 th March, 2020-31st May, 2020, unlock 0.1 and 0.2, as well as weekly lockdown in July, 2020 including night curfew and implementation of effective quarantine periods for travelers. (a) $S I_{S} I_{N} R$ model based on the novel stochastic approach $(\beta$ $\left.=0.15625 ; R_{0}=1.25\right)$. (b) $S I_{S} I_{N} R$ model based on the novel stochastic approach $\left(\beta=0.1875 ; R_{0}=1.5\right)$. (c) $S I_{S} I_{N} R$ model based on the novel stochastic approach $\left(\beta=0.25 ; R_{0}=2.0\right)$. (d) $S I_{S} I_{N} R$ model based on the novel stochastic approach $(\beta$ $\left.=0.3125 ; R_{0}=2.5\right)$. (e) $\operatorname{SI}_{S} I_{N} R$ model based on the novel stochastic approach $\left(\beta=0.375 ; R_{0}=3.0\right)$. Note: The parameters such as S, I, A and R are used to express susceptible, infected (symptomatic), infected (asymptomatic) and recovered patients, respectively

Tamil Nadu showed the least case fatality rate in the country. A large local cluster in Koyambedu of Chennai was identified in May. The case growth rate in Chennai saw a dip in July. Contact-tracing, testing and surveillance model was applied by the government to prohibit the spread of the novel COVID-19 in Tamil Nadu. The state is gifted with 85 laboratories approved by Indian Council of Medical Research (ICMR), capable of conducting tests. The state witnessed lockdown from 25th March, 2020. This lockdown was relaxed to an extent from 04th May, 2020 onwards. Furthermore, the government decided to extend the lockdown until 30th June, 2020. Under the lockdown, significant relaxations were made from 01st June, 2020. A stricter lockdown was enforced in the four most affected districts including Chennai and its three neighbouring districts of Chengalpattu, Thiruvallur and Kancheepuram from 19 
to 30th June, 2020 (The Hindu, 2020b; The Indian Express, 2020a).

The number of daily new cases dropped from 2,400 in the first week of July to 1,013 on the 31 th July, 2020. Recovery rate improved from 60 to $85 \%$. The city's positivity rate stood around $9 \%$ at the end of July. The total of infected people reported on 31 st July, 2020 is 99,714 , consisting of 2,110 deaths and 84,916 recoveries (The Indian Express, 2020b; The New Indian Express, 2020).

The suitable graphs have been plotted using the above inputs to discuss the spread of the novel COVID-19 pandemic in the Chennai region of the Tamil Nadu state (Fig. 22) on the basis of the values of ' $R_{0}$ ' using stochastic mathematical models based on the modified $S I_{S} I_{N} R$ transmission network model. The peaks of infected cases (both symptomatic and asymptomatic cases) as well as the end of SARSCoV-2 pandemic are attended soon after 31 st July, 2020 with an increase in the values of ' $\beta$ ' and ' $R_{0}$ '. For Chennai city, the first case $\left(\beta=0.15625 ; R_{0}=\right.$ $1.25)$ is more appropriate in current scenario as it shows the peak of the novel COVID-19 pandemic in December, 2020 (Fig. 22a). The curve shows the zero case of pandemic by the end of March, 2021.

\section{Stochastic Model Forecasting the Outbreak of the Novel COVID-19 in Ahmedabad}

As the novel COVID-19 cases increase in the state government of Gujarat partially locked down four cities - Ahmedabad, Surat, Vadodara and Rajkot. Ahmedabad has reported 25 cases (Rajkot has ten, Vadodara, Surat and Gandhinagar have nine cases each, Bhavnagar has six cases, GirSomnath has two cases and Kutch, Mehsana and Porbandar have one case each). In total, six coronavirus positive patients have died in the state till the end of March, 2020. Till March end five patients have been discharged, the officials declared, two are on ventilator support. Out of the total 73 patients, 32 had a foreign travel history, 37 contracted it through local transmission and 4 had interstate travel history. Every store selling nonessential goods in the four cities saw shut down till 25th March, 2020. As part of the mitigatory measures, only half strength was operating at government offices to be on a rotational basis till 29th March, 2020. Honourable Chief Minister announced the new Civil hospital of Ahmedabad for treating patients infected with the coronavirus. This hospital has a 1,200-bed capacity. With over 3,000 COVID-19 cases and 149 deaths, Ahmedabad was the second city after Mumbai in number of cases and in deaths (The Wire, 2020; India Today, 2020c).

Ahmedabad in Gujarat was announced as a hotspot of the novel COVID-19 by Government of India as 3 patients out of total 5 cases in the city had died. The Government of India also declared that Dilshad Garden and Nizamuddin in Delhi, Noida and Meerut in Uttar Pradesh, Bhilwara in Rajasthan, Kasargod and Pathanamthitta in Kerala, Mumbai and Pune in Maharashtra as the hotspots. The city alone accounts for nearly $65 \%$ of total cases and $70 \%$ of deaths in Gujarat. The total number of infected people reported on $31 \mathrm{st}$ July, 2020 is 25,080 , consisting of 1,152 deaths and 20,618 recoveries (The Hindu, 2020c; Amdavad Municipal Corporation, 2020).
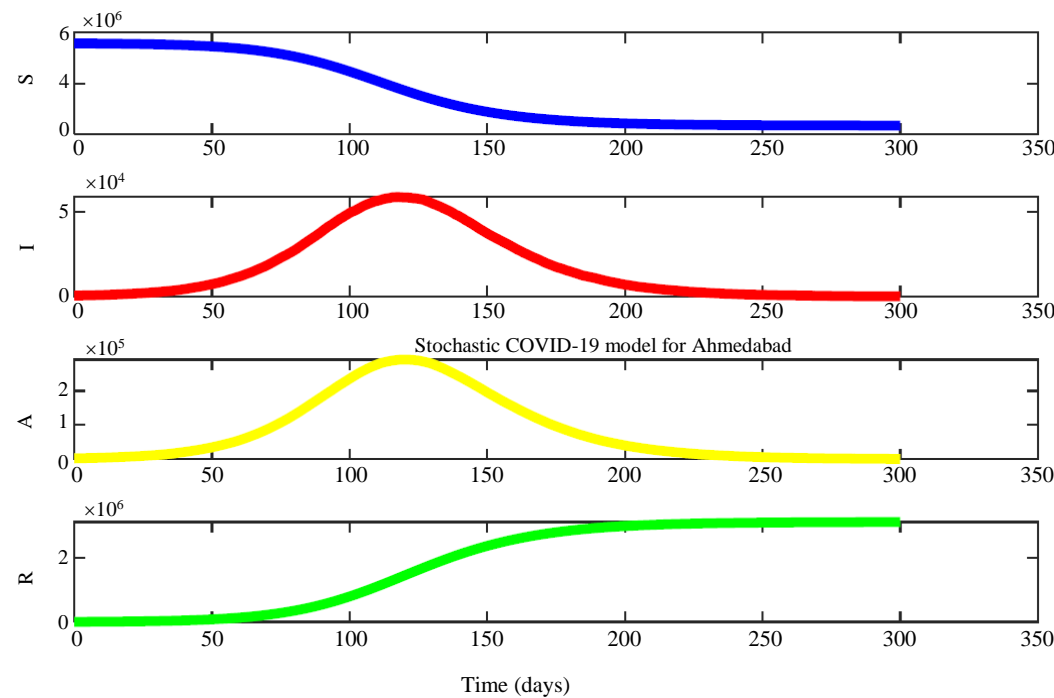

(a) 
Eva Gupta et al. / American Journal of Infectious Diseases 2020, 16 (4): 135.170 DOI: 10.3844/ajidsp.2020.135.170
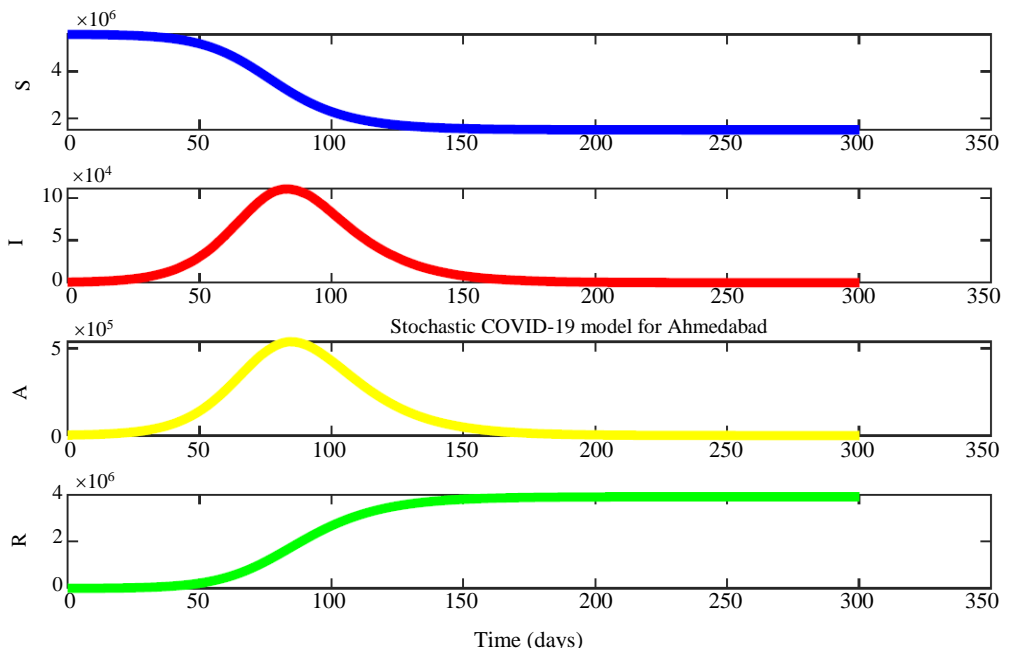

(b)
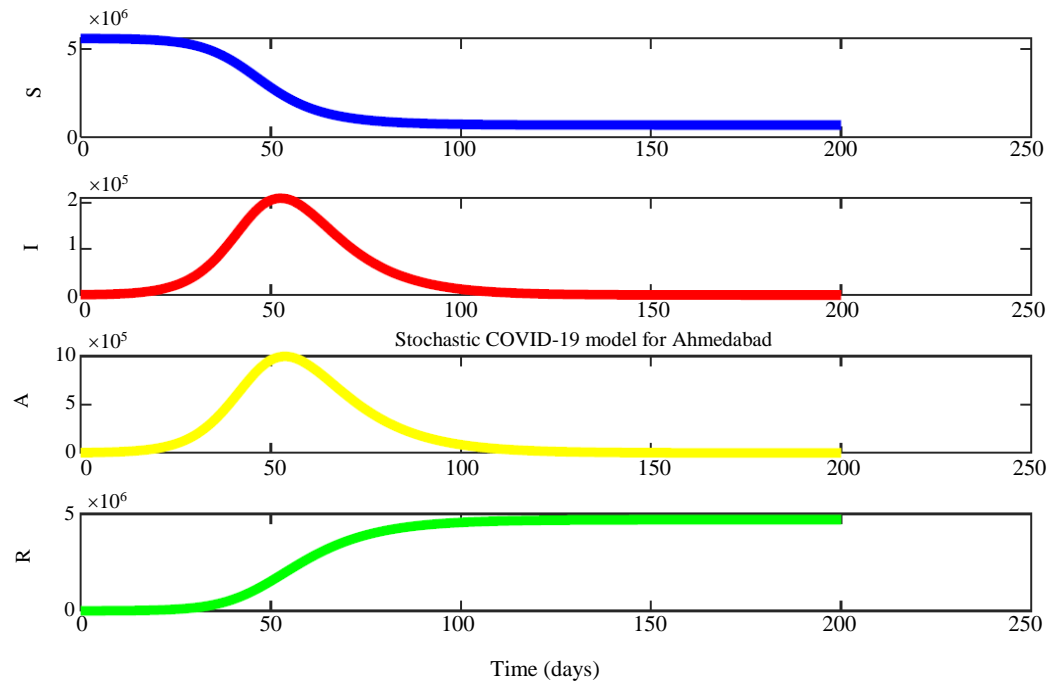

(c)
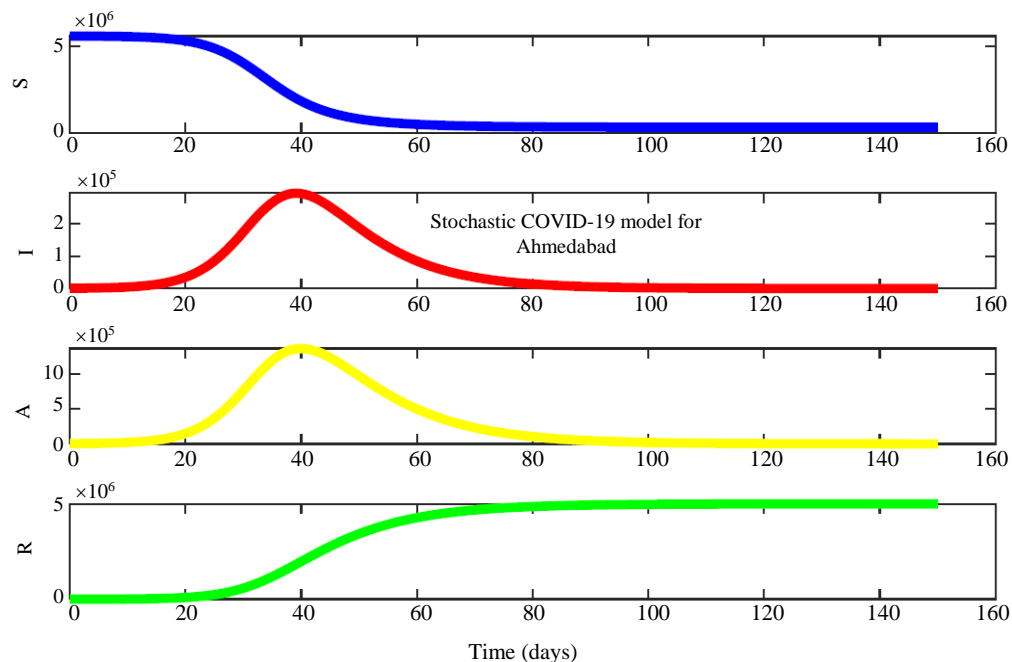

(d) 

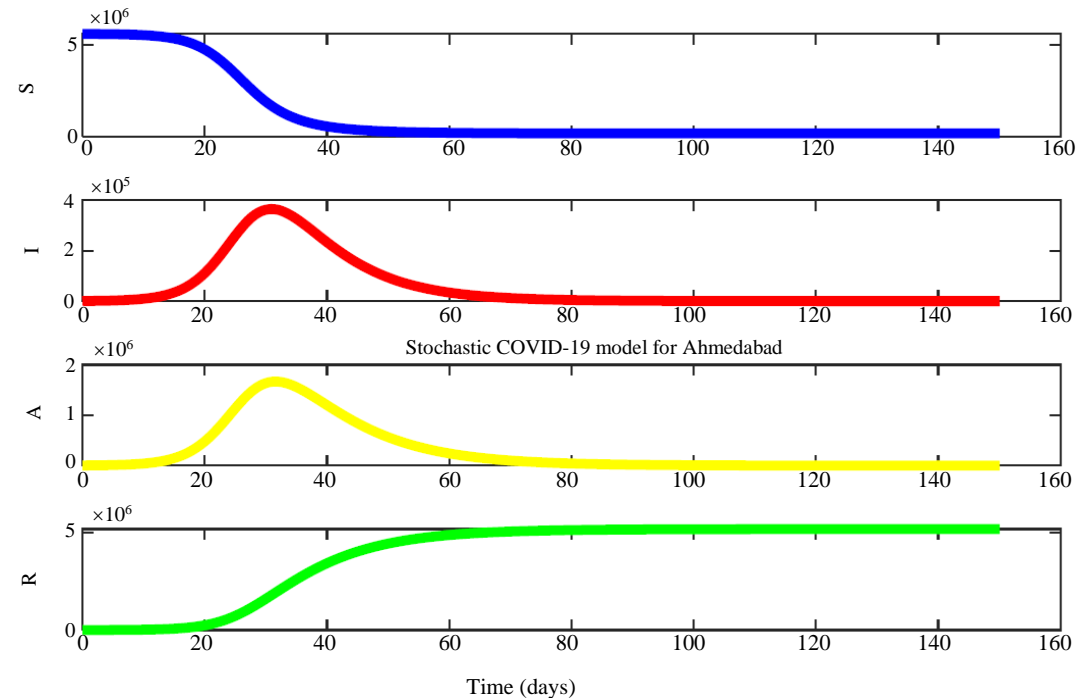

(e)

Fig. 23: The stochastic $S I_{S} I_{N} R$ model of the novel COVID-19 for Ahmedabad considers a sustained lockdown of 61 days, i.e., 25th March, 2020-31st May, 2020, unlock 0.1 and 0.2, as well as weekly lockdown in July, 2020 including night curfew and implementation of effective quarantine period for travelers. (a) $S I_{S} I_{N} R$ model based on the novel stochastic approach $(\beta=$ $\left.0.15625 ; R_{0}=1.25\right)$. (b) $S I_{S} I_{N} R$ model based on the novel stochastic approach $\left(\beta=0.1875 ; R_{0}=1.5\right)$. (c) $S I_{S} I_{N} R$ model based on the novel stochastic approach $\left(\beta=0.25 ; R_{0}=2.0\right)$. (d) $S I_{S} I_{N} R$ model based on the novel stochastic approach $(\beta=0.3125$; $\left.R_{0}=2.5\right)$. (e) $S_{S} I_{N} R$ model based on the novel stochastic approach $\left(\beta=0.375 ; R_{0}=3.0\right)$. Note: The parameters such as $\mathrm{S}, \mathrm{I}$, $\mathrm{A}$ and $\mathrm{R}$ are used to express susceptible, infected (symptomatic), infected (asymptomatic) and recovered patients, respectively

The suitable graphs have been plotted using the above inputs to discuss the spread of the novel COVID19 pandemic in the Ahmedabad region of the Gujarat state (Fig. 23) on the basis of the values of ' $R_{0}$ ' using stochastic mathematical models based on the modified $S I_{S} I_{N} R$ transmission network model. The peaks of infected cases (both symptomatic and asymptomatic cases) as well as the end of SARS-CoV-2 pandemic are attended soon after 31st July, 2020 with an increase in the values of ' $\beta$ ' and ' $R_{0}$ '. Ahmedabad city is expected to attend the novel COVID-19 pandemic peak in December, 2020 (Fig. 23a); first case $\left(\beta=0.15625 ; R_{0}=1.25\right)$ is more appropriate in current scenario). The curve shows the zero case of pandemic by the end of March, 2021.

\section{Discussion}

The aim of a mathematical model is not to make unconditional claims about the consequences of interventions, but to reveal the relation between assumptions and outcomes. The motive of our investigation through effective simulation of the modified susceptible $(S)$-infected (symptomatic) $\left(I_{S}\right)$ infected (asymptomatic) $\left(I_{N}\right)$-Recovered $(R)$ i.e., $S I_{S} I_{N} R$ transmission network model is to restrict the chain of transmission of the novel COVID-19 pandemic in India.

We developed the compartmental susceptible $(S)$ infected (symptomatic) $\left(I_{S}\right)$-infected (asymptomatic) $\left(I_{N}\right)$ - recovered $(R)$ i.e., $S I_{S} I_{N} R$ model. The efficacy and potential of base, education, vaccination and education and vaccination programs are established with the published datasets through a validation studies as well under both stochastic and deterministic cases using similar inputs for the meningitis outbreak case in University of Central Florida (UCF) campus. The results are found in fairly good agreement with the published datasets.

We have considered various scenarios by varying the basic reproduction numbers from mean to extremes using base, education, vaccination and education and vaccination models for effective estimation about the peak and end of the pandemic to assist in the revision and effective implementation of health policies as mitigatory measures in India. Initially, we predicted the progress of the pandemic deterministically for a range of values of the basic reproduction number, ' $R_{0}$ ', based on certain assumptions. We observed that the results are frightening.

\section{Summary}

For $R_{0}=1.25$, we conclude to attend the peak of the novel COVID-19 pandemic by the end of December, 2020 , with around $6 \%$ of the total population being infected at that time. For $R_{0}=1.5$, we conclude to attend the peak of the novel COVID-19 pandemic by the end of November, 2020, with around $12 \%$ of the 
total population being infected at that time. For $R_{0}=$ 2.0 , we conclude to attend the peak of the novel COVID-19 pandemic by the end of October, 2020, with around $21 \%$ of the total population being infected at that time. For $R_{0}=2.5$, we conclude to attend the peak of the novel COVID-19 pandemic in mid-September, 2020, with around $29 \%$ of the total population being infected at that time. For $R_{0}=3.0$, we conclude to attend the peak of the novel COVID-19 pandemic by the end of August, 2020 , with around $37 \%$ of the total population being infected at that time. We did rigorous analyses to inculcate general assumptions and strategies through contact tracing based on the values of contact ratio operated for the basic reproduction numbers, ' $R_{0}$ '. The analysis with $R_{0}=1.25$ corresponding to $\beta=0.15625$; fits best under the current scenario $\beta=0.15625$.

We initially investigated a base model by considering the mean of the basic reproduction number, ' $R_{0}$ '. Later on, the investigation is done to study the effect of educating people regarding maintaining social distancing and personal hygiene which we called as education model. Here, we found out that by educating people we may extend the progress and diminish the number of infected at a particular time, which can potentially help healthcare services to manage the patients. The effect of mass vaccination combined with the education model is investigated herewith and concluded with fact that the novel COVID-19 pandemic is predicted to end after 100 days from 15 th June, 2021 based on the value of ' $\beta$ ' around 0.128125 (Fig. 18). The prediction is done on the basis of inputs based on assumptions and expert suggestions such as (a) availability of the vaccine to be available by the end of February, 2021 and (b) $61 \%$ of the population of India would be vaccinated up to $15^{\text {th }}$ June, 2021. The deterministic analyses based on the modified $S I_{S} I_{N} R$ transmission network model are concluded with the facts such as (a) the symptomatic cases are found to be around $80 \%$ less than asymptomatic cases and (b) the peaks of infected cases (both symptomatic and asymptomatic cases) as well as end of SARS-CoV-2 pandemic are estimated soon after 31st July, 2020 in December, 2020 (Fig. 11-18) and by the end of March, 2021, respectively.

Furthermore, we stochastically predicted the progress of the pandemic in the top five majorly affected cities in India which includes Delhi, Pune, Mumbai, Chennai and Ahmedabad. The prediction about the transmission of the novel COVID-19 outbreak is done using a range of values of the basic reproduction number, ' $R_{0}$ ', as 1.25 , $1.5,2.0,2.5$ and 3.0 based on the general assumptions and strategies through contact tracing. We could reduce the value of the basic reproduction number, ' $R_{0}$ ', by educating people regarding social distancing and personal hygiene or by implementing the complete or partial lockdown across India or across majorly affected cities to diminish the social contacts which will eventually reduce the spread of the disease. For all majorly affected top five cities, the first case $(\beta=$ $\left.0.15625 ; R_{0}=1.25\right)$ is more appropriate as it shows the peak of the novel COVID-19 pandemic in December, 2020 (Fig. 20a). The pandemic curves show the zero case of pandemic by the end of March, 2021.

\section{Acknowledgment}

The idea behind was to study efficient epidemiological models for simulating the transmissibility of the novel coronavirus disease 2019 (COVID-19) outbreak across the nation and understand the effective mitigatory measures to break the transmission chain of the pandemic in India.

\section{Author's Contributions}

Eva Gupta: Research Scholar, Amity University, Uttar Pradesh, India (An Institute of National Importance), is pursuing $\mathrm{PhD}$ in Electrical Engineering. She has reviewed papers on above topic and developed MATLAB programs for simulating the outbreak of the novel COVID-19 pandemic in India using deterministic and stochastic approaches.

Nand Jee Kanu: Research Scholar, S. V. National Institute of Technology, Surat, India (An Institute of National Importance), is pursuing $\mathrm{PhD}$ in Mechanical Engineering. He has reviewed papers on above topic and developed MATLAB programs for simulating the outbreak of the novel COVID-19 pandemic in India using deterministic and stochastic approaches.

Amartya Munot: BE Research Scholar, Mechanical Engineering, S. A. P. D. J. Pathshala's Walchand Institute of Technology, Solapur, India. He has analyzed inputs through MATLAB programs to study the outbreak of SARS-CoV-2 pandemic in India using deterministic and stochastic approaches.

Venkateshwara Sutar: BE Research Scholar, Mechanical Engineering, JSPM Narhe Technical Campus, Pune, India. He has analyzed inputs through MATLAB programs to study the outbreak of SARSCoV-2 pandemic in India using deterministic and stochastic approaches.

Umesh Kumar Vates: Associate Professor, Amity University, Uttar Pradesh, India, has completed his $\mathrm{PhD}$ in Mechanical engineering from IIT (ISM) Dhanbad, India (An Institute of National Importance). He has investigated and supervised the research work to predict the transmission of SARS-CoV-2 pandemic in India.

Gyanendra Kumar Singh: Associate Professor, Mechanical Design and Manufacturing Engineering, Adama Science and Technology University, Adama, Ethiopia, has completed his $\mathrm{PhD}$ in Mechanical 
engineering from M. N. National Institute of Technology, Allahabad, India (An Institute of National Importance). He has investigated and supervised the research work to predict the transmission of SARS-CoV2 pandemic in India.

\section{Conflict of Interest}

The authors declare that there are no conflicts of interest.

\section{Planning and Motivation}

We reviewed the relevant articles on the novel coronavirus (SARS-CoV2) published online within the past few months with the aim to join the battle against coronavirus disease (COVID-19).

\section{Data Availability}

The authors confirm that the data supporting the findings of this study are available within the article.

\section{Ethics}

The author does not see any ethical issues that may arise after the publication of this manuscript.

\section{Appendix A}

Singh and Adhikari (2020) reviewed the modified age-structured Susceptible-Infected-Recovered (SIR) model of the novel COVID-19 outbreak in India by considering the activity of the Indian government to execute mitigatory measures such as physical distancing, lockdown in India to restrict the spread of global pandemic across the nation. They considered the birth and death rates to be the same within a specific age group. Depending upon the real-time time-independent data, mitigatory social distancing and age groups, the basic Reproduction number $\left(R_{o}\right)$ was assessed by using the modified age-structured SIR model.

The people are categorized in various ages such as ' $M$ ' groups denoted as $i=1,2, \ldots M$. The people under a specific age were described as ' $i$ ' and they are categorized into susceptible patients $\left(S_{i}\right)$, symptomatic patients $\left(I_{i}^{s}\right)$, asymptomatic patients $\left(I_{i}^{a}\right)$ and removed patients $\left(R_{i}\right)$. Therefore, the total number of people within a specified age group $(i)$ will be sum of all these categories as $N_{i}=S_{i}+I_{i}^{a}+I_{i}^{s}+R_{i}$.

Each $N_{i}$ was denoted as the total number of people as shown in Equation (3):

$$
N=\sum_{i=1}^{M} N_{i}
$$

The transmission rate of the novel COVID-19 in susceptible patients within a specific age group $(i)$ is represented by using Equation (4):

$\lambda_{i}(t)=\beta \sum_{j=1}^{M}\left(C_{i j}^{a} \frac{I_{j}^{a}}{N_{j}}+C_{i j}^{s} \frac{I_{j}^{s}}{N_{j}}\right), i, j=1, \ldots M$

where, ' $\beta$ ' term was the chance of transmission of the pandemic due to physical contact and a $C_{i j}^{a}$ and $C_{i j}^{s}$ were used to represent the number of cases of social contact between asymptomatic patients within the age group $(j)$ and susceptible patients within an age group (i), respectively. The term ' $\gamma$ ' is also known as curing rate, was not dependent on age and was the same for both asymptomatic (ratio, $\alpha$ ) and symptomatic patients (ratio, $\bar{\alpha}=1-\alpha)$.

The pandemic growth rate was expressed using agestructured SIR model in Equation (5):

$\left.\begin{array}{l}\dot{S}_{i}=-\lambda_{i}(t) S_{i}, \\ \dot{I}_{i}^{a}=\alpha \lambda_{i}(t) S_{i}-\gamma I_{i}^{a}, \\ \dot{I}_{i}^{s}=\bar{\alpha} \lambda_{i}(t) S_{i}-\gamma I_{i}^{s}, \\ \dot{R}_{i}=\gamma\left(I_{i}^{a}+I_{i}^{s}\right) .\end{array}\right\}$

The age group of the persons was expressed as the ratio of $N_{i} / N$ and social contact was expressed using $C_{i j}^{a}$ and $C_{i j}^{s}$ matrices. Furthermore, the symptomatic patients were supposed to have less contact as compared to the asymptomatic patients and thus, $C_{i j}^{s}=f C_{i j}^{a} \equiv f C_{i j}$, (where, $0 \leq f \leq 1)$.

The people were expected to remain in homes, office places, schools and other places for self-isolation, therefore the contact matrix represented by using Equation (6):

$C_{i j}=C_{i j}^{H}+C_{i j}^{W}+C_{i j}^{S}+C_{i j}^{O}$

For a specific population size, the contact matrix was represented as $N_{i} C_{i j}=N_{j} C_{i j}$.

On a large basis, mitigatory social distancing was modeled and expressed as a time functions $u^{W}(t), u^{S}(t)$ and $u^{O}(t)$ and thus Equation (7) shows the timedependent contact matrix:

$C_{i j}(t)=C_{i j}^{H}+u^{W}(t) C_{i j}^{W}+u^{S}(t) C_{i j}^{S}+u^{o}(t) C_{i j}^{O}$.

The physical and social distancing were imposed effectively across the country during lockdown and thus a single household contact function was represented by using the linear expression (8): 
$2 u(t)=-\tanh \left(\frac{t-t_{\text {on }}}{t_{w}}\right)+\tanh \left(\frac{t-t_{\text {off }}}{t_{w}}\right)$

The basic reproductive number was evaluated from null COVID-19 spots (where $S_{i}=N_{i}$ ) and infected persons were expressed using $2 M \times 2 M$ matrix, as shown in Equation (9):

$J=\gamma(L-1)$.

The $2 M \times 2 M$ generation matrix is obtained from Equation (10):

$L=\left(\begin{array}{ll}L^{a a} & L^{a s} \\ L^{s a} & L^{s s}\end{array}\right)$

where,

$$
L_{i j}^{a a}=\frac{\alpha \beta}{\gamma} C_{i j}^{a} \frac{N_{i}}{N_{j}}, L_{i j}^{a s}=\frac{\alpha \beta}{\gamma} C_{i j}^{s} \frac{N_{i}}{N_{j}}
$$

$L_{i j}^{s a}=\frac{\alpha \beta}{\gamma} C_{i j}^{a} \frac{N_{i}}{N_{j}}, L_{i j}^{s s}=\frac{\bar{\alpha} \beta}{\gamma} C_{i j}^{s} \frac{N_{i}}{N_{j}}$

The identity matrix, $\bar{I}(2 M \times 2 M)$ was used to encompass both asymptomatic and symptomatic patients as shown in Equation (11):

$\bar{I}=\left(I^{a}, I^{s}\right)=\left(I_{1}^{a}, \ldots, I_{M}^{a}, I_{1}^{s}, \ldots I_{M}^{s}\right)$

The dynamics of the above Equation (11) was expressed using Equation (12):

$$
\bar{I}(t)=\exp [\gamma(L-1) t] \bar{I}(0)
$$

where, ' $L$ ' was used to evaluate the eigenvectors, ' $V$ ' and the diagonal matrix of eigenvalues, $\Lambda=\operatorname{diag}\left(\Lambda_{1}, \ldots\right.$, $\left.\Lambda_{1 M}\right)$ to calculate the expression, $\exp [\chi(L-1) t]=V$ diag $\exp [\exp \chi(\Lambda-1) t] V^{-1}$.

For pandemic growth, the span $\mathrm{L}$ must be greater than one and thus the basic reproduction number, ' $R_{0}$ ', is expressed as Equation (13):

$$
R_{0} \equiv \rho(L)=\max \left\{\left|\Lambda_{1}\right|, \ldots,\left|\Lambda_{1 M}\right|\right\} .
$$

The basic reproductive number was reasonably good to support the spread of the pandemic if the eigenvalue was real as shown in Equation (14):

$$
\exp \left[\gamma\left(R_{0}-1\right) t\right]
$$

The basic reproductive number was based on (a) the chance of transmission of disease by social contact, (b) the social contact matrix, (c) the ratio of persons without and with symptoms of SARS-CoV-2 and (d) the ratio of symptomatic patients who were in self-isolation. The conversions such as (a) and (b) were done to transform the formulation into its linear form for time-based stability matrix. The time-based stability matrix, was expressed using Equation (15):

$R_{0}^{e f f}(t) \equiv \rho\left(L^{(t)}\right)=\max \left\{\left|\Lambda_{1}^{(t)}\right|, \ldots,\left|\Lambda_{1 M}^{(t)}\right|\right\}$

The cases of infected patients at any time were expressed as shown in Equation (16):

$\bar{I}(t+\delta t)=\exp \left[\gamma\left(L^{(t)}-1\right) \delta t\right] \bar{I}(t)$

The basic reproductive number played a significant role, when the eigenvalue was real for its highest magnitude for the tremendous growth of the pandemic in a very short time. The end of the pandemic was shown with the rise and fall of the spread of SARS-CoV-2 outbreak were shown with time constants.

\section{Appendix B}

\section{Calculations for the base Model}

$R=$ Radius of infection $=0.00001 \mathrm{~km}$

$q=$ Probability of infective transmitting the infection $=$ 0.1

$\rho=$ Population density $=382$ number of people per square $\mathrm{km}$

$\bar{v}=$ Average traffic speed $=263.270 \mathrm{~km} /$ day

$\beta=$ Contact ratio

$\beta=\frac{8 R q \bar{v} \rho}{\pi}$

$\beta=\frac{8 \times 0.00001 \times 0.1 \times 263.270 \times 382}{\pi}$

$\beta=0.2560$

Calculations for Education and Education and Vaccination Models

$R=$ Radius of infection $=0.00001 \mathrm{~km}$

$q=$ Probability of infective transmitting the infection $=$ 0.5

$\rho=$ Population density $=382$ number of people per square $\mathrm{km}$

$\bar{v}=$ Average traffic speed $=263.270 \mathrm{~km} /$ day

$\beta=$ Contact ratio

$\beta=\frac{8 R q \bar{v} \rho}{\pi}$

$\beta=\frac{8 \times 0.00001 \times 0.5 \times 263.270 \times 382}{\pi}$

$\beta=0.128125$ 


\section{Values of Contact Ratio for Different Scenarios}

$R=$ Radius of infection $=0.00001 \mathrm{~km}$

$q$ = Probability of infective transmitting the infection $=$ 0.5

$\rho=$ Population density $=382$ number of people per square $\mathrm{km}$

For, $\bar{v}=$ average traffic speed $=160.626 \mathrm{~km} /$ day; $\beta=$ 0.1562

$\bar{v}=$ Average traffic speed $=192.751 \mathrm{~km} /$ day; $\beta=$ 0.1875

For, $\bar{v}=$ average traffic speed $=251.712 \mathrm{~km} /$ day; $\beta=$ 0.2512

$\bar{v}=$ Average traffic speed $=321.252 \mathrm{~km} /$ day $; \beta=$ 0.3125

For, $\bar{v}=$ average traffic speed $=385.521 \mathrm{~km} /$ day; $\beta=$ 0.3752

\section{References}

Amdavad Municipal Corporation. (2020). COVID-19 Ahmedabad city. Amdavad Municipal Corporation. https://ahmedabadcity.gov.in/portal/index.jsp.

Atkins, T. (2010). Using modeling and simulation to evaluate disease control measures.

BBC News. (2020a). Coronavirus vaccine: when will we have one? BBC News. Available: https://www.bbc.com/news/health-51665497

BBC News. (2020b). Coronavirus vaccine trial begun by drug firms GSK and Sanofi. BBC News. https://www.bbc.com/news/business-54009633

Bhola, J., Venkateswaran, V. R., \& Koul, M. (2020). Corona Epidemic in Indian context: Predictive Mathematical Modelling. medRxiv.

Business Today. (2020). Coronavirus: Delhi govt. announces Operation SHIELD to take on COVID$19 . \quad$ Business Today. https://www.businesstoday.in/latest/trends/coronavir us-delhi-govt-announces-operation-shield-to-takeon-COVID-19/story/400648.html

Chang, S. L., Harding, N., Zachreson, C., Cliff, O. M., \& Prokopenko, M. (2020). Modelling transmission and control of the COVID-19 pandemic in Australia. arXiv preprint arXiv:2003.10218.

Chatterjee, K., Chatterjee, K., Kumar, A., \& Shankar, S. (2020). Healthcare impact of COVID-19 epidemic in India: A stochastic mathematical model. Medical Journal Armed Forces India.

COVID-19 India. (2020). Novel Coronavirus-India. COVID-19 India. https://www.COVID19india.org/

Daughton, A. R., Generous, N., Priedhorsky, R., \& Deshpande, A. (2017). An approach to and webbased tool for infectious disease outbreak intervention analysis. Scientific reports, 7, 46076.
Deb, S., \& Majumdar, M. (2020). A time series method to analyze incidence pattern and estimate reproduction number of COVID-19. arXiv preprint arXiv:2003.10655.

Dhama, K., Sharun, K., Tiwari, R., Dadar, M., Malik, Y. S., Singh, K. P., \& Chaicumpa, W. (2020). COVID19 , an emerging coronavirus infection: advances and prospects in designing and developing vaccines, immunotherapeutics and therapeutics. Human vaccines $\&$ immunotherapeutics, 1-7.

Dhanwant, J. N., \& Ramanathan, V. (2020). Forecasting COVID 19 growth in India using SusceptibleInfected-Recovered (SIR) model. arXiv preprint arXiv:2004.00696.

Gupta, E., \& Kanu, N. J. (2020). An Insight into the Simplified RP Transmission Network, Concise Baseline and SIR Models for Simulating the Transmissibility of the Novel Coronavirus Disease 2019 (COVID-19) Outbreak. American Journal of Infectious Diseases.

Hindustan Times. (2020a). India most infected by the novel COVID-19 among Asian countries, leaves Turkey behind. Hindustan Times. https://www.hindustantimes.com/india-news/indiamost-infected-by-COVID-19-among-asiancountries-leaves-turkey-behind/storyJjd0AqIsuL3yjMWg29uJ3I.html

Hindustan Times. (2020b). The novel COVID-19: Number of recoveries exceeds active cases for first time. Hindustan Times. https://www.hindustantimes.com/indianews/COVID-19-number-of-recoveries-exceedactive-cases-for-first-time/storyuA0C6zESJTdk19UQ21Hc2M.html

India Today. (2020a). COVID-19: Another reason why unlock 1.0 may be ill-timed in India. India Today. https://www.indiatoday.in/newsanalysis/story/COVID-19-unlock-1-0-indialockdown-coronavirus-cases-1687162-2020-06-09

India Today. (2020b). Pune woman tests positive for COVID-19, had returned from Netherlands. Maharashtra total now at 43. India Today. https://www.indiatoday.in/india/story/pune-womantests-positive-for-COVID-19-had-returned-fromnetherlands-maharashtra-total-now-at-42-16570642020-03-18

India Today. (2020c). Coronavirus: Gujarat government declares lockdown rules for shops, firms. India Today. https://www.indiatoday.in/india/story/coronavirusgujarat-govt-declares-lockdown-rules-for-shopsfirms-1658305-2020-03-21

Inventiva. (2020a). A vaccine which could be helpful in curing coronavirus. Inventiva. https://www.inventiva.co.in/stories/inventiva/avaccine-which-could-be-helpful-in-curingcoronavirus/ 
Inventiva. (2020b). ICMR to get about 7 lakhs rapid antibody testing kits to India by April 8. Inventiva. https://www.inventiva.co.in/trends/riyarana/icmr-toget-about-7-lakhs-rapid-antibody-testing-kits-toindia-by-april-8/

Kumar, S. (2020). Predication of Pandemic COVID-19 situation in Maharashtra, India. medRxiv.

Livemint. (2020). Coronavirus lockdown extended till 31 May, says NDMA. Livemint. https://www.livemint.com/news/india/COVID-19lockdown-4-0-coronavirus-lockdown-extended-till31-may-says-ndma-11589715203633.html

Mackolil, J., \& Mahanthesh, B. (2020). Mathematical Modelling of Coronavirus disease (COVID-19) Outbreak in India using Logistic Growth and SIR Models.

Mandal, S., Bhatnagar, T., Arinaminpathy, N., Agarwal, A., Chowdhury, A., Murhekar, M., ... \& Sarkar, S. (2020). Prudent public health intervention strategies to control the coronavirus disease 2019 transmission in India: A mathematical model-based approach. The Indian journal of medical research, 151(2-3), 190.

Mazumder, A., Arora, M., Bharadiya, V., Berry, P., Agarwal, M., Behera, P., ... \& Parameswaran, G. G. (2020). SARS-CoV-2 epidemic in India: epidemiological features and in silico analysis of the effect of interventions. F1000Research, 9.

NDTV. (2020). 2,347 COVID cases in Maharashtra in $24 \mathrm{~h}$, Mumbai crosses 20,000-mark. NDTV. https://www.ndtv.com/india-news/2-347-newcoronavirus-cases-in-maharashtra-in-24-hoursmumbai-crosses-20-000-mark-2230403

News18. (2020) How the govt. machinery acted quickly to spread awareness about coronavirus. News 18 . https://www.news18.com/news/opinion/how-thegovt-machinery-acted-quickly-to-spread-awarenessabout-novelCOVID-19-2537029.html

Pai, C., Bhaskar, A., \& Rawoot, V. (2020). Investigating the dynamics of COVID-19 pandemic in India under lockdown. Chaos, Solitons \& Fractals, 138, 109988.

Rajesh, A., Pai, H., Roy, V., Samanta, S., \& Ghosh, S. (2020). CoVID-19 prediction for India from the existing data and SIR (D) model study. medRxiv.

Ramola, R. C. (2020). Projected Spread of COVID-19 through India Based on SIR Model. Reproduction, $1-52$.

Ranjan, R. (2020). Predictions for COVID-19 outbreak in India using Epidemiological models. medRxiv.

Rihan, F. A., \& Anwar, M. N. (2012). Qualitative analysis of delayed SIR epidemic model with a saturated incidence rate. International Journal of Differential Equations, 2012.

Sahasranaman, A., \& Kumar, N. (2020). Network structure of COVID-19 spread and the lacuna in India's testing strategy. Available at SSRN 3558548 .
Singh, R., \& Adhikari, R. (2020). Age-structured impact of social distancing on the COVID-19 epidemic in India. arXiv preprint arXiv:2003.12055.

The Hindu. (2020a). Curbs put on roadside shops, outlets, eateries in Navi Mumbai. The Hindu. https://www.thehindu.com/news/national/coronaviru s-live-updates-march-19-2020/article31105138.ece

The Hindu. (2020b). Three fresh cases detected in Tamil Nadu. The Hindu. https://www.thehindu.com/news/national/coronaviru s-live-updates-march-24-2020/article31149240.ece

The Hindu. (2020c). Coronavirus Ahmedabad records over 3,000 cases, 149 deaths. The Hindu. https://www.thehindu.com/news/national/otherstates/coronavirus-ahmedabad-records-over-3000cases-149-deaths/article31475675.ece

The Hindustan Times. (2020a). Pune needs to avoid mistakes Mumbai made to get through COVID-19. The Hindustan Times. https://www.hindustantimes.com/pune-news/puneneeds-to-avoid-mistakes-mumbai-made-to-getthrough-COVID-19/storysGYIt2yMrg6Ns3diWRH1cJ.html

The Hindustan Times. (2020b). Maharashtra facing surge in cases even outside red zones. The Hindustan Times. https://www.hindustantimes.com/indianews/maharashtra-facing-surge-in-cases-evenoutside-red-zones/storyKIx5rXJHDr8xVpEg9HW9MO.html

The Indian Express. (2020a). Lockdown begins in Chennai, 3 districts today; surge in cases. The Indian Express.

https://indianexpress.com/article/india/lockdownbegins-in-chennai-3-districts-today-surge-in-cases6465698/

The Indian Express. (2020b). Over 35\% Tamil Nadu coronavirus cases linked to Koyambedu market in Chennai. The Indian Express. https://indianexpress.com/article/india/over-35-tncases-linked-to-a-market-in-chennai-6408861/

The New Indian Express. (2020). The novel COVID-19: ray of hope as daily cases in Chennai dip closer to three-digit mark. The New Indian Express. https://www.newindianexpress.com/cities/chennai/2 020/jul/31/covid-19-ray-of-hope-as-daily-cases-inchennai-dip-closer-to-three-digit-mark2177383.html

The Times of India. (2020a). Coronavirus: 15 lakh who flew back to India between Jan 18-Mar 23 under lens. The Times of India. https://timesofindia.indiatimes.com/india/15-lakhwho-flsew-back-to-india-between-jan-18-mar-23under-lens/articleshow/74855553.cms 
The Times of India. (2020b). Manish Sisodia lauds locals for making 'Operation SHIELD' successful. The Times of India. https://timesofindia.indiatimes.com/city/delhi/manis h-sisodia-lauds-locals-for-making-operation-shieldsuccessful/articleshow/75200268.cms

The Wire. (2020). Government identifies 10 coronavirus hotspots, testing to be boosted. The Wire. https://thewire.in/government/coronavirus-hotspotsindia-testing-boost

Times Network. (2020). Overview of the novel COVID19 - an India perspective. Times Network. https://www.timesnownews.com/india/photogallery/overview-of-the novel COVID-19-an-indianperspective-an-insightful-report-by-timesnetwork/584153
Tiwari, A. (2020). Modelling and analysis of COVID-19 epidemic in India. medRxiv.

Wikipedia. (2020a). The novel COVID-19 pandemic in India. https://en.wikipedia.org/wiki/the novel COVID-19_pandemic_in_India

Wikipedia. (2020b). Basic reproduction number. Wikipedia.

https://en.wikipedia.org/wiki/Basic_reproduction_nu mber

Wikipedia. (2020c) The novel COVID-19 pandemic in Delhi. Wikipedia. https://en.wikipedia.org/wiki/the novel COVID-19_pandemic_in_Delhi. 